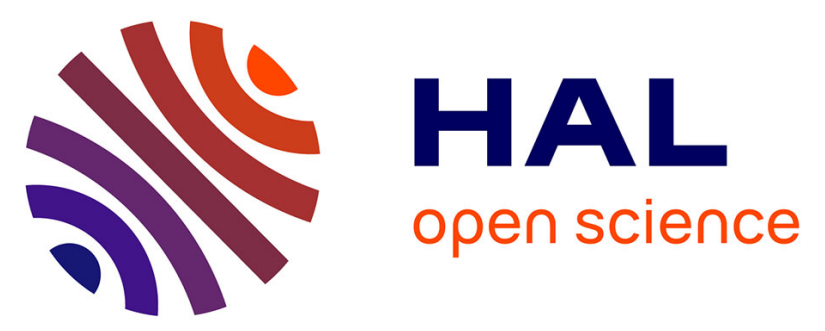

\title{
Four-way coupled Eulerian-Lagrangian Direct Numerical Simulations in a vertical laminar channel flow
} Jonathan Schillings, Olivier Léon Doche, Mauricio Tano Retamales, Frédéric Bauer, Jonathan Deseure, Sedat F. Tardu

\section{- To cite this version:}

Jonathan Schillings, Olivier Léon Doche, Mauricio Tano Retamales, Frédéric Bauer, Jonathan Deseure, et al.. Four-way coupled Eulerian-Lagrangian Direct Numerical Simulations in a vertical laminar channel flow. International Journal of Multiphase Flow, 2017, 89, pp.92-107. 10.1016/j.ijmultiphaseflow.2016.10.006 . hal-02545878

\section{HAL Id: hal-02545878 https://hal.science/hal-02545878}

Submitted on 28 Apr 2020

HAL is a multi-disciplinary open access archive for the deposit and dissemination of scientific research documents, whether they are published or not. The documents may come from teaching and research institutions in France or abroad, or from public or private research centers.
L'archive ouverte pluridisciplinaire HAL, est destinée au dépôt et à la diffusion de documents scientifiques de niveau recherche, publiés ou non, émanant des établissements d'enseignement et de recherche français ou étrangers, des laboratoires publics ou privés. 


\section{Four-way coupled Eulerian-Lagrangian Direct Numerical Simulations in a vertical laminar channel flow}

Jonathan Schillings ${ }^{1}$, Olivier Doche ${ }^{2}$, Mauricio Tano Retamales ${ }^{3}$, Frédéric Bauer $^{2}$, Jonathan Deseure ${ }^{1}$, Sedat Tardu ${ }^{4}$

${ }^{1}$ Univ. Grenoble Alpes, LEPMI, F-38000 Grenoble, France

${ }^{2}$ Univ. Grenoble Alpes, SIMaP, F-38000 Grenoble, France

${ }^{3}$ IN2P3 - Univ. Grenoble Alpes, LPSC, F-38000 Grenoble, France

${ }^{4}$ Univ. Grenoble Alpes, LEGI, F-38000 Grenoble, France

\section{Abstract}

Direct Numerical Simulations of laminar two-phase flows in a vertical channel are investigated. An Eulerian-Lagrangian approach allows tracking each bubble position with a fourway coupling strategy, i.e. taking into account bubble $\Leftrightarrow$ fluid and bubble-bubble interactions. The flow configuration has been chosen to highlight the buoyancy effects due to significant values of void fraction (high numbers of bubbles); hence the bubbles collisions and wall effects are the critical parameters to ensure the dispersion of the bubble plume. The DNS approach is self-consistent and does not rely on empirical correlations for the bubbles collective dynamic or closure relations. It is found that the DNS predicts well the behavior of the bubble plume and its back effect on the liquid phase when compared with a mixture model and experimental data. The elastic nature of collisions, the sensitivity of the mean and RMS values of velocities and of the void fraction to the mesh quality together with the evolution of wall shear-rate are explored. All simulations are run on a standard workstation by means of an effective numerical parallelization. 


\section{1- Introduction}

Accurate simulations of two-phase flows require sophisticated numerical techniques such as the Direct Numerical Simulations (DNS). DNS have originally been developed for investigating turbulent flows by reproducing and solving all the interactions between the motion scales with no regards to time averaged or space averaged operations, hence without having the need of closure models (such as the model RANS, $k-\varepsilon$, etc.). Huge quantitative information are obtained through DNS that can legitimately be classified as numerical experiments (Jiménez, 2003). There is a strong analogy between turbulent and dispersed twophase flows of moderately dense suspensions (i.e. void fraction greater than 0.01), at least from a numerical simulation point of view. Both are three dimensional time-evolving flows and exhibit similar nonlinear complexity arising from the advection terms of the Navier-Stokes equations. In addition, perturbations of the carrier phase can be generated by the back effect of the transported dispersed phase, which could be combined (or not) with the local instability of the flow itself in the case of turbulence. The carrier phase induced perturbations can be identified and analyzed in a way similar to fluctuations occurring in a single-phase turbulent flow and analogous statistical post-treatment can be applied to the large data generated by DNS of multiphase flows. It is without saying that additional numerical and modeling complexities arise in multiphase flows coming from the interaction between phases and the choice of an appropriate coupling strategy is therefore crucial.

Various strategies for modeling two-fluid flows can be found in the literature and correspond to different levels of complexity and accuracy (e.g. Ishii, 1987; Zhang and Prosperetti, 1994). Yet, attempts to resolve dispersed flows by DNS arose quite recently. At this point, it is worth noting that the two-phase flow equations can be resolved in two different ways. In the first case each particle trajectory is individually monitored and calculated by means of the Newton's second law, and the carrier fluid represented by the Navier-Stokes equations is treated as a continuous phase (i.e. a Lagrangian-Eulerian approach, e.g. Boivin et al., 1998). In the second case, the two-phase flow system equations is expressed as the interaction between two continuous fluid: the dispersed and continuous phase (called two-fluid or Eulerian-Eulerian model) (Delnoij et al., 1997; Esmaeeli and Tryggvason, 1999). The two fluids model provides 
certainly the most detailed picture of the particles behavior and, since each particle geometry is fully resolved, allows a fine description of the interactions between the particles and the particles with the continuous phase. Hence, this approach could be used to catch the surface deformation of bubbles, the induced perturbation of the flow field, the effect of surface tension, and/or clarify the coalescence phenomena, etc. However, tracking the interface requires a dynamic meshing around moving boundaries (Lu et al., 2006; Tryggvason et al., 2001) and since only a moderate amount of inclusions (classically a few hundred) can be computed, it cannot be yet representative of a typical industrial process. For the case of the Eulerian-Lagrangian approach, each element composing the discrete phase is treated as point-like (Laín et al., 2002; Nierhaus et al., 2007), in the sense that their geometrical properties (shapes, size) are implicitly taken into account in the formulation of the interfacial forces. During the last two decades, these models have widely been used to investigate the effects of particle transport on the turbulent field characteristics (e.g. Berg et al., 2006; Mazzitelli et al., 2003). Among those works, one can distinguish between studies neglecting the discrete phase effects on the flow (i.e. oneway coupling), and approaches taking into account their back reactions via a dedicated term expressed in the momentum equation of the continuous phase (i.e. two way coupling strategy) (Druzhinin and Elghobashi, 2001; Squires and Eaton, 1990).

The applications are numerous and become more and more crucial for our industrial partners since bubbly flows are found in several transformation processes (bubble columns for phase extractions, electrolysis processes for hydrogen production, etc.). So they turned to be a classical subject for various academic studies in the chemical engineering domain (e.g. Akita and Yoshida, 1974; Lapin and Lübbert, 1994). In the singular case of electrochemical conversion which is mainly employed in metal production such as aluminum production or to gas production such as chlorine production, bubbly flows have severed effects on the operation of electrochemical cell. It is well known that the efficiency of the classical electrolysis processes depends strongly on the mass transfer at the electrode surface.

Additionally, a flow can be induced by the electrochemical bubble production and it has for instance been shown that the electrochemical reactions can be enhanced by pumped electrolyte flow (Schillings et al., 2015). Hence, whatever the modeling strategy is and since 
performing generic DNS is still beyond our capabilities, it is clear that the most challenging point is now to identify a way to predict adequately the collective effects of bubbles. Indeed, to the authors knowledge, only closure terms derived from a few and dedicated experimental data can be found in the literature (e.g. Leighton and Acrivos, 1987; Nicolai et al., 1995). Without any reliable stand-alone formulation for the collective effect modeling, only qualitative results can be gained from two-phase flows numerical approaches, as can be found for example in Schillings et al. (2015) or Dahlkild (2001). Therefore, a finite difference code developed by our team for turbulent simulation (Bauer et al., 2015) is the keystone to the present DNS of twophase flows with four-way coupling.

In the present paper, we report the study of a bubbly flow in a vertical channel as it can be found in a typical electrolysis cell for hydrogen production. The two-phase flow resolution and its coupling methodology are then detailed and applied to a vertical channel where the amount of gaseous product leads to a significant effect on the continuous phase velocity. We investigate an Eulerian-Lagrangian model in order to simulate the dispersed two-phase flow in a regime that would be laminar without the presence of the discrete phase. The main objective of the paper is to take into account the four-way coupling which is essential in such configuration because no wall normal bubble plume dispersion can occur without bubble-bubble interactions.

\section{2- Numerical model}

In this paper, the dynamic of an electrochemically generated two-phase flow is investigated through a straight Eulerian-Lagrangian formulation. The choice of illustrating the four-way coupling strategy in a typical electrolysis cell is motivated by the fact that it combines not only experimental data and modeling works in the literature but also fits totally to a practical (i.e. industrial) application for which the DNS approach can be fully justified.

The electrolyte motion is described by the well-known time dependent and threedimensional Navier-Stokes equations under assumptions of a Newtonian and incompressible liquid as the continuous phase. The isothermal condition is also assumed since it can be shown that the temperature rise due to the electrochemical reactions is negligible against the two- 
phase buoyancy effect in our operating conditions. Gas bubbles trajectories are calculated by resolving the Newton's second law for each of them. As stated before, the resolution is performed with a four-way coupling (i.e. accounting bubble-bubble and bubble $\Leftrightarrow$ liquid interactions), including the effect of the wall on the bubbles trajectories. The configuration used throughout this work is depicted in Fig. 1. Both electrodes constituting the lateral channel walls are set vertically in order to generate buoyancy forces in the streamwise direction, $\boldsymbol{x}$. The two electrodes are located at $y=0$ and $y=2 h$, start at $x=0$ and end at $x=L_{x}$. Their size along the $\boldsymbol{z}$ direction is infinite in order to simulate pseudo-2D cases. In this work, the liquid phase is restricted to the laminar regime.

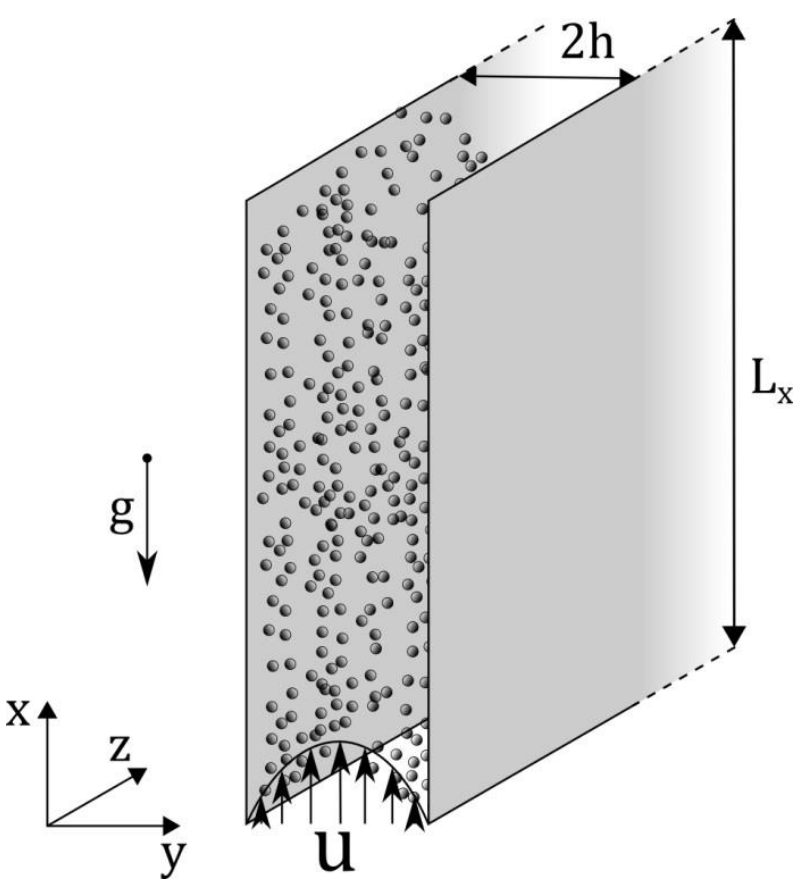

Fig. 1: Scheme of the electrodes and flow configuration.

Numerical simulations are performed with a validated finite difference code developed by our team (Bauer et al., 2015; Doche et al., 2013, 2012). This code, optimized for turbulent simulations, handles massive parallel computing and fits the requirement for strong flow couplings with large number of inclusions.

The DNS data are compared to the simulations of a homogenous (also called mixture) model here. This model considers the gas-liquid mixture as a unique continuous Newtonian fluid ruled by a momentum conservation equation and a volume conservation equation. Both 
equations depend on the void fraction which is a scalar variable representing the local proportion of dispersed phase in the mixture volume. The evolution of the void fraction is resolved by means of the relative flux, a phenomenological closure term extracted from theoretical and empirical studies that specifies the superficial velocity of the dispersed phase relatively to the mixture motion. The complete mixture model formulation can be found in our previous work (Schillings et al., 2015).

The following subsections detail the numerical models resolved for each phase and their interactions.

\section{1 - Continuous phase}

The continuous phase is resolved by Direct Numerical Simulation (DNS) of the NavierStokes equations in the following form:

$$
\begin{gathered}
\boldsymbol{\nabla} \cdot \boldsymbol{u}=0 \\
\frac{\partial \boldsymbol{u}}{\partial t}+(\boldsymbol{u} \cdot \boldsymbol{\nabla}) \boldsymbol{u}+\frac{1}{\rho_{C}} \nabla p-v_{C} \nabla^{2} \boldsymbol{u}=\frac{1}{\rho_{C}} \boldsymbol{f}_{\text {ext }}
\end{gathered}
$$

Here, $\rho_{C}$ and $v_{C}$ are the constant density and kinematic viscosity, $\boldsymbol{u}$ is the velocity field and $p$ the pressure of the continuous phase. $\boldsymbol{f}_{\text {ext }}$ is the force field traducing the action of the bubbles on the liquid. The boundary conditions applied in the entire work are as follow: a no-slip condition is applied at $y=0$ and $y=2 h=L_{y}$ and periodicity applies at $z=0$ and $z=L_{z}$, where $L_{z}$ is the width of the numerical domain only, as it is recalled that this direction is supposedly infinite. A standard Poiseuille profile is imposed at $x=0$ and the flow leaves the domain with radiative open boundary conditions (i.e. without stress) at $x=L_{x}$. Lengths, velocities and times are nondimensionalized respectively by the semi inter-electrode gap $h$, the maximum Poiseuille velocity $u_{C P}$ and the characteristic time $\frac{h}{u_{C P}}$. The variables given in their non-dimensional form are noted with the exponent *. The Reynolds number is defined as $R e_{h}=\frac{u_{C P} h}{v_{C}}$, which is different from the usual one based on the mean velocity and the hydraulic diameter. 


\section{2 - Discrete phase}

As stated previously, the study of electrochemically generated two-phase flows is of great interest due to the huge impact of bubble plumes on the hydrodynamic, species transport and charge transfer. For practical reasons, the referential case studied in this work is the production of hydrogen and oxygen by alkaline water electrolysis, which allows some assumptions. In this process, bubbles diameter is less than $100 \mu \mathrm{m}$ so that they can be considered as solid light particles without internal circulation and coalescence (Janssen et al., 1984). In addition, a no-slip condition holds at bubble surface due to common interface contamination. The bubble production rate is directly proportional to a controllable electrolysis current density. The physics of bubble growth and detachment are not taken into account here and the particles are added directly in the domain in the vicinity of the electrodes with zero velocity. The following section details the numerical approach used to compute the bubbles motions, the bubble-bubble interactions and the wall collisions.

\subsection{1 - Equation of motion and hydrodynamic force}

The bubble motion is resolved by a Lagrangian tracking through the resolution of the Newton's second law:

$$
\rho_{D} \frac{d \boldsymbol{v}}{d t}=\rho_{D} \boldsymbol{g}+\boldsymbol{F}_{C-D}+\boldsymbol{F}_{c o l}
$$

Where $\rho_{D}$ is the density of the dispersed phase, $\boldsymbol{v}$ the bubble velocity, $\boldsymbol{g}$ the gravitational acceleration field, $\boldsymbol{F}_{C-D}$ the hydrodynamic volume force and $\boldsymbol{F}_{c o l}$ is a sum of forces arising from eventual collisions with other bubbles or a wall.

The hydrodynamic force exerted on a rigid sphere moving in an unsteady Stokes flow has been formulated by Maxey and Riley (1983) and Gatignol (1983) and consists in the integration of external stresses on a sphere surface. The hydrodynamic force can be expressed as the sum

of two distinct contributions, $\boldsymbol{F}_{C-D, a}$ and $\boldsymbol{F}_{C-D, b}$. The first one includes the pressure and viscous stress effects on the undisturbed flow that would occupy the volume of the sphere: 


$$
\boldsymbol{F}_{C-D, a}=-\rho_{C} \boldsymbol{g}+\rho_{C} \frac{D \boldsymbol{u}}{D t}
$$

Secondly, the force $\boldsymbol{F}_{C-D, b}$ arises from flow perturbations caused by the presence of the inclusion itself and is commonly expressed as the sum of various terms known as the added mass, the drag and the history (also called Basset) terms. The Maxey-Riley/Gatignol relation is now the reference formulation; however it should be adapted for our studying case. Primarily, the Faxen terms - i.e. second-order correction terms depending on $\nabla^{2} \boldsymbol{u}$ - can be neglected because of the small bubble size compared to the characteristic flow length, and the Basset history term is of little influence in bubbly flows while necessitating additional computational efforts (Domgin et al., 1998). In addition, the Maxey-Riley/Gatignol formulation is obtained only for creeping flow conditions. Effects from small but finite particle Reynolds number $R e_{D}=$ $\frac{2 r\left\|\boldsymbol{V}_{\boldsymbol{r}}\right\|}{v_{C}}$ must be taken into account, with $r$ the radius of the sphere and $\boldsymbol{V}_{\boldsymbol{r}}=\boldsymbol{v}-\boldsymbol{u}$ its relative velocity. Those are principally the drag and lift corrections. Finally, the near-wall effects on the drag and lift terms are included. Equation (5) summarizes the different terms of the hydrodynamic force due to the disturbance flow:

$$
\boldsymbol{F}_{C-D, b}=\boldsymbol{F}_{\text {add }}+\boldsymbol{F}_{\text {drag }}+\boldsymbol{F}_{\text {lift }}
$$

Each term is detailed in the next subsections.

\subsection{2 - The virtual, or added, mass term}

Boussinesq (1885) introduced an expression for the transient hydrodynamic force in which appeared for the first time the virtual mass, expressed in its Lagrangian form in Eq. (6):

$$
\boldsymbol{F}_{\text {add }}=\frac{1}{2} \rho_{C}\left(\frac{d \boldsymbol{v}}{d t}-\frac{D \boldsymbol{u}}{D t}\right)
$$

This term is due to the acceleration of a volume of the surrounding fluid along with the sphere, which was found to be equivalent to half the volume of the sphere.

\subsection{3 - The drag effects}

As stated by Arcen et al. (2006), many expressions of the drag force can be found and the better choice depends on the type of application aimed by the model. In the case of an 
electrochemical gas production - which is a surface bubble generation similar to nucleation the wall proximity should play a role on the hydrodynamic force.

$$
\boldsymbol{F}_{\text {drag }}=-\frac{3 C_{d}}{8 r} \rho_{C} \mathcal{C}_{\|, \perp}\left\|\boldsymbol{V}_{\boldsymbol{r}}\right\| \boldsymbol{V}_{\boldsymbol{r}}
$$

Here, $C_{d}=\frac{24}{R e_{D}}\left(1+\frac{3}{16} R e_{D}\right)$ is the drag coefficient as proposed by Oseen (1910), which is less restrictive than the Stokes drag coefficient. Oseen derived this coefficient theoretically for small but finite values of the particle Reynolds number while the Stokes coefficient is correct for vanishing $R e_{D}$. The Oseen correction shows very good precision for $R e_{D} \leq 0.4$ and an error of only $7.5 \%$ at $R e_{D}=1$. This is therefore valid in many applications including the present bubbly flows with gas inclusions smaller than $100 \mu \mathrm{m}$. Simulations performed in typical electrolysis cases show that only $1 \sim 2 \%$ of the bubbles are associated to a Reynolds number larger than 0.4 , with an average value of 0.55 . Other attempts to develop theoretical results at higher Reynolds numbers exist, but they diverge rapidly when used out of their range of application. To cover larger ranges of $R e_{D}$, one can also use empirical relations such as that of Schiller and Naumann (1935), which fits experimental data for $R e_{D}<10^{5}$. In this work, the Oseen's formula is preferred because of its analytical nature.

$$
\mathcal{C}_{\|, \perp}=\left(\begin{array}{ccc}
C_{\|} & & \\
& C_{\perp} & \\
& & C_{\|}
\end{array}\right) \text {is a correction of the drag coefficient which includes the presence }
$$

of the wall in the flow perturbation.

$$
\begin{gathered}
C_{\|}=\left(1-\frac{9}{16} \kappa+\frac{1}{8} \kappa^{3}-\frac{45}{256} \kappa^{4}-\frac{1}{16} \kappa^{5}\right)^{-1} \\
C_{\perp}=\left(1-\frac{9}{8} \kappa+\frac{1}{2} \kappa^{3}\right)^{-1}
\end{gathered}
$$

where $\kappa=\frac{r}{l}$ and $l$ is the distance of the sphere center to the nearest wall. The first correction (8) is extracted from the work of Faxén (1923) and is found to be very close to the analytical result for $\frac{r}{l}<0.76$. Eq. (9) can be found in the work of Wakiya (1960). It is an approximation precise to $O\left(\omega^{3}\right)$ of the exact expression developed in infinite series by Brenner (1961). 


\subsection{4 - The lift force}

In a wall-bounded flow, bubbles dynamics are affected by the shear of the base flow. The shear rate near the wall can be significantly higher than that of a classical Poiseuille flow, if the buoyancy induced by the discrete phase is strong. Saffman (1965) showed that particles translating and rotating with respect to a uniform shear flow are subjected to a lift force perpendicular to the flow direction. The Saffman lift force holds for unbounded flows, small values of $R e_{D}$ and $R e_{\gamma}=\frac{4 r^{2}|\dot{\gamma}|}{v_{C}}$ and for $R e_{D} \ll R e_{\gamma}{ }^{2}$ where $R e_{\gamma}$ is the Reynolds number based on the shear and $\dot{\gamma}=\frac{\partial u_{x}}{\partial y}$ is the main shear rate of the flow.

In the present work, we use the optimum lift force of Wang et al. (1997) that combines many results found in the literature (Cox and Hsu, 1977; Vasseur and Cox, 1977; McLaughlin, 1991, 1993; Cherukat and Mclaughlin, 1994). It is the most complete formulation of the lift force and can be applied in every flow region. The expression is highly dependent on characteristic non-dimensional parameters such as the Stokes lengthscale $L_{S}=\frac{v_{C}}{\left|V_{r, x}\right|}$, the Saffman lengthscale $L_{\gamma}=\sqrt{\frac{\overline{v_{C}}}{|\dot{\gamma}|}}$, the ratio $\epsilon=\operatorname{sign}\left(\dot{\gamma} V_{r, x}\right) \frac{L_{S}}{L_{\gamma}}$ and the dimensionless distance from the wall $l^{\gamma}=\frac{l}{L_{\gamma}}$. The complete formulation of the optimum lift force is detailed in Annexe 1.

\subsection{5 - Collisions, elasticity and lubrication models}

Hoomans et al. (1996) presented a discrete particle model developed for twodimensional simulations of fluidized-bed. They implemented a sequence to simulate the collisions of particles, which will be shortly presented in §3.2.3. They used a hard sphere model for the collisions, which assumes impulsive (i.e. instantaneous point forces) collisions and the conservation of the spherical shape after collision. The particles are in contact at the moment of collision, but dissipation occurs so that the kinetic energy of the particles is not completely conserved during the impact. Following this work, Delnoij et al. (1997) simulated dispersed gasliquid flows with the same collision sequence and a hard sphere model. However, a thin liquid film resists the approach of two bubbles and hence the repelling occurs without direct contact between the spheres. They approximated the bubbles radius of influence by $r \sqrt[3]{1+C_{a d d}}$ where 
$C_{a d d}=0,5$ is the added mass coefficient. In addition, they considered elastic collisions, i.e. a total restitution of the bubbles kinetic energy after rebound.

In order to understand the choices made in the present work, some clarifications about collisions and lubrication are needed.

Different cases potentially occur when two spheres approach each other (all the observations detailed here are valid for a sphere approaching a wall). A direct contact occurs if the fluid resistance is negligible, i.e. if the viscous stress growing inside the fluid when escaping the narrowing gap is small compared to the inertia of the sphere. During contact, strain energy can be stored in the spheres in the form of very small elastic deformations, and this is the release of this energy that causes the impulse of rebound and a quick return to a spherical shape: this is called an elastic collision. Yet, various losses such as plastic deformations, internal viscous dissipation or remaining vibrations might occur, depending on the nature of the continuous and the dispersed phases. The collision is thus inelastic and the kinetic energy of the spheres before impact is not fully restored. In that case, a restitution coefficient is defined as $0 \leq \varepsilon_{r}=-\frac{v_{\text {reb }}}{\boldsymbol{v}_{\text {app }}} \leq 1$ where $\boldsymbol{v}_{\text {app }}$ is the approach velocity, i.e. the relative velocity of the spheres along the line defined by the centers of masses, and $\boldsymbol{v}_{r e b}$ the velocity after rebound. One might notice that this formulation of $\varepsilon_{r}$ is a simplification for identical spheres.

In the case of an important fluid resistance, the pressure that builds up in the thin volume of fluid during the gap diminution generates a reaction force on the spheres. This force can be calculated by the lubrication theory (Hocking, 1973) which holds for $\frac{h_{l}}{2 r} \ll 1$ and $R e_{a p p} \frac{h_{l}}{2 r} \ll 1$, where $h_{l}$ is the distance between the surfaces of the spheres and $R e_{a p p}=$ $\frac{\left\|v_{a p p}\right\| 2 r}{v_{C}}$ is the Reynolds number based of the approach velocity. The reaction force, equal in magnitude but opposite in sign for the two spheres, being inversely proportional to the interspace height and proportional to the approach velocity makes the direct contact impossible. Davis et al. (1986) claimed that the pertinent characteristic number in elastohydrodynamic collisions is the Stokes number $S t=\frac{1}{9} \frac{\rho_{D}}{\rho_{C}} R e_{a p p}$ which is a ratio of the 
particle inertia on the fluid viscous forces. For very small values of the Stokes number, the inertia of the particles is not sufficient to induce a high pressure increase in the fluid layer and their kinetic energy is dissipated by viscosity. For high inertia particles, two cases are likely: completely rigid spheres do not rebound and the kinetic energy is dissipated in the fluid, while elastic spheres eventually rebound. The same definition of the restitution coefficient holds to take into account inelastic deformations, internal viscous dissipation, remaining vibrations or dissipation in the fluid (Gondret et al., 2002).

The important fluid resistance and inelastic collisions hypotheses hold here for the bubbly flows of interest. Collisions are thus instantaneous and occur at a distance $\frac{h_{l}}{r}=0.1$ due to lubrication with a zero restitution coefficient, both for bubble-bubble and wall-bubble collisions. The collision force $\boldsymbol{F}_{\text {col }}$ from Eq. (3) is not explicitly calculated but instead the bubble velocity is directly corrected after a collision (see 3.2.3).

\section{3- Numerical details}

\section{1 - Continuous phase}

\subsection{1 - Time advancement procedure}

The solution at the next time iteration $k+1$ is obtained by integrating the Eq. (2) over the $t$ to $t+\Delta t$ interval. This equation is solved in several steps according to the well-known fractional step approach which ensures the divergence-free condition (Eq. (1)) of the velocity field at each time iteration. The temporal integration of Eq. (2) is written as:

$$
\int_{t}^{t+\Delta t} \frac{\partial \boldsymbol{u}}{\partial t} d t=-\frac{1}{\rho_{C}} \int_{t}^{t+\Delta t} \boldsymbol{\nabla} P d t-\frac{1}{\rho_{C}} \int_{t}^{t+\Delta t} \boldsymbol{\nabla} p^{\prime} d t-\int_{t}^{t+\Delta t}(\boldsymbol{u} \cdot \boldsymbol{\nabla}) \boldsymbol{u} d t+v_{C} \int_{t}^{t+\Delta t} \nabla^{2} \boldsymbol{u} d t-\frac{1}{\rho_{C}} \int_{t}^{t+\Delta t} \boldsymbol{f}_{\text {ext }} d t
$$

where $\nabla P$ is the mean spatial pressure gradient, $\nabla p^{\prime}$ is the pressure gradient fluctuation. Thus, by defining $\boldsymbol{u}^{k}$ and $\boldsymbol{u}^{k+1}$ the velocity fields at a time $t$ and $t+\Delta t$, the temporal advancement Eq. (10) can be expressed as:

$$
\boldsymbol{u}^{k+1}=\boldsymbol{u}^{k}+\boldsymbol{T}_{\text {pmean }}+\boldsymbol{T}_{\text {pfluc }}+\boldsymbol{T}_{a d v}+\boldsymbol{T}_{\text {diff }}+\boldsymbol{T}_{\text {fext }}
$$


with:

$$
\begin{gathered}
\boldsymbol{T}_{\text {pmean }}=-\frac{1}{\rho_{C}} \int_{t}^{t+\Delta t} \boldsymbol{\nabla} P d t \\
\boldsymbol{T}_{\text {pfluc }}=-\frac{1}{\rho_{C}} \int_{t}^{t+\Delta t} \nabla p^{\prime} d t \\
\boldsymbol{T}_{\text {adv }}=-\int_{t}^{t+\Delta t}(\boldsymbol{u} \cdot \boldsymbol{\nabla}) \boldsymbol{u} d t \\
\boldsymbol{T}_{\text {diff }}=v_{C} \int_{t}^{t+\Delta t} \nabla^{2} \boldsymbol{u} d t \\
\boldsymbol{T}_{\text {fext }}=-\frac{1}{\rho_{C}} \int_{t}^{t+\Delta t} \boldsymbol{f}_{\text {ext }} d t
\end{gathered}
$$

Without external force term the mean pressure gradient term $\boldsymbol{T}_{\text {pmean }}$ is evaluated with an Euler method (first order), and is calculated by applying the flow conservation constraint on the whole domain at the current time iteration. The coupling strategy will be further discussed in section 3.3. Advection and diffusion terms, respectively $\boldsymbol{T}_{a d v}$ and $\boldsymbol{T}_{\text {diff }}$ are estimated explicitly from velocity fields obtained from the previous $k-1$ and current time step $k$. It is worth noting the possibility of using implicit schemes to improve the stability of the diffusion terms, but at the cost of expensive multiband matrix inversions and a loss of genericity. The term $\boldsymbol{T}_{p f l u c}$ is the fluctuating pressure gradient evaluated from the pressure at $k+1$ for sake of stability (Bauer et al., 2015). Eq. (11) can be reformulated as:

$$
\boldsymbol{u}^{k+1}=\widetilde{\boldsymbol{u}}^{k+1}-\frac{\Delta t}{\rho_{C}} \boldsymbol{\nabla} p^{\prime k+1}
$$

where $\tilde{\boldsymbol{u}}^{k+1}=\boldsymbol{u}^{k}+\boldsymbol{T}_{\text {pmean }}+\boldsymbol{T}_{a d v}+\boldsymbol{T}_{\text {diff }}$ is a first estimation of the velocity fields, based on terms known at the current time iteration. $p^{\prime k+1}$ is then calculated by applying the divergence free operator to Eq. (13) and by solving the Poisson equation: 


$$
\nabla^{2} p^{\prime k+1}=\frac{\rho_{C}}{\Delta t} \boldsymbol{\nabla} \cdot \widetilde{\boldsymbol{u}}^{k+1}
$$

In the present study, all calculations are realized with a third order Runge-Kutta time advancement scheme. That does not modify the sequence of resolution but divides the main time step into three substeps, each one being expressed with a dedicated set of coefficients.

\subsection{2 - Spatial discretization}

Spatial numerical operators are expressed by using an explicit optimized (EO) finite differentiation scheme at the fourth order (Bauer et al., 2015). EO schemes are derived from the dispersion-relation-preserving (DRP) schemes developed by Tam and Webb (1993). Unlike compact schemes, explicit schemes use only the values of a function the neighboring points in order to approximate the derivatives. Hence, approximation of the derivatives is direct while it necessarily implies a matrix inversion for compact schemes, which is generally a more expensive operation from a numerical point of view. It is well known that standard explicit schemes require fine grid resolution to ensure a good precision. To compensate, the optimization in the EO schemes consists of adding complementary terms to the Taylor development of the derivatives in order to minimize the error in the Fourier space, with the largest wavenumber range possible.

The computational domain of size $L_{x} \times L_{y} \times L_{z}$ has an associated mesh of $N_{x} \times N_{y} \times N_{z}$ nodes.

\section{2 - Discrete phase}

\subsection{1 - Volume distribution}

As underlined previously, the Eulerian-Lagrangian formulation approximates the inclusions by point elements and the reaction force exerted by each element is applied at the position of its center of mass. This approximation is correct for Eulerian grids much coarser than the inclusions size but becomes instable when the computational cell size is comparable to or smaller than the inclusions size. This issue is overcome by distributing the volume of bubble and the reaction force among the numerical cells it actually occupies. A scheme of the volume 
distribution process is presented in Fig. 2. The illustration shows on the left-hand side the affected cells if the bubbles are defined by their center of mass only and with a volume distribution strategy on the right-hand side.
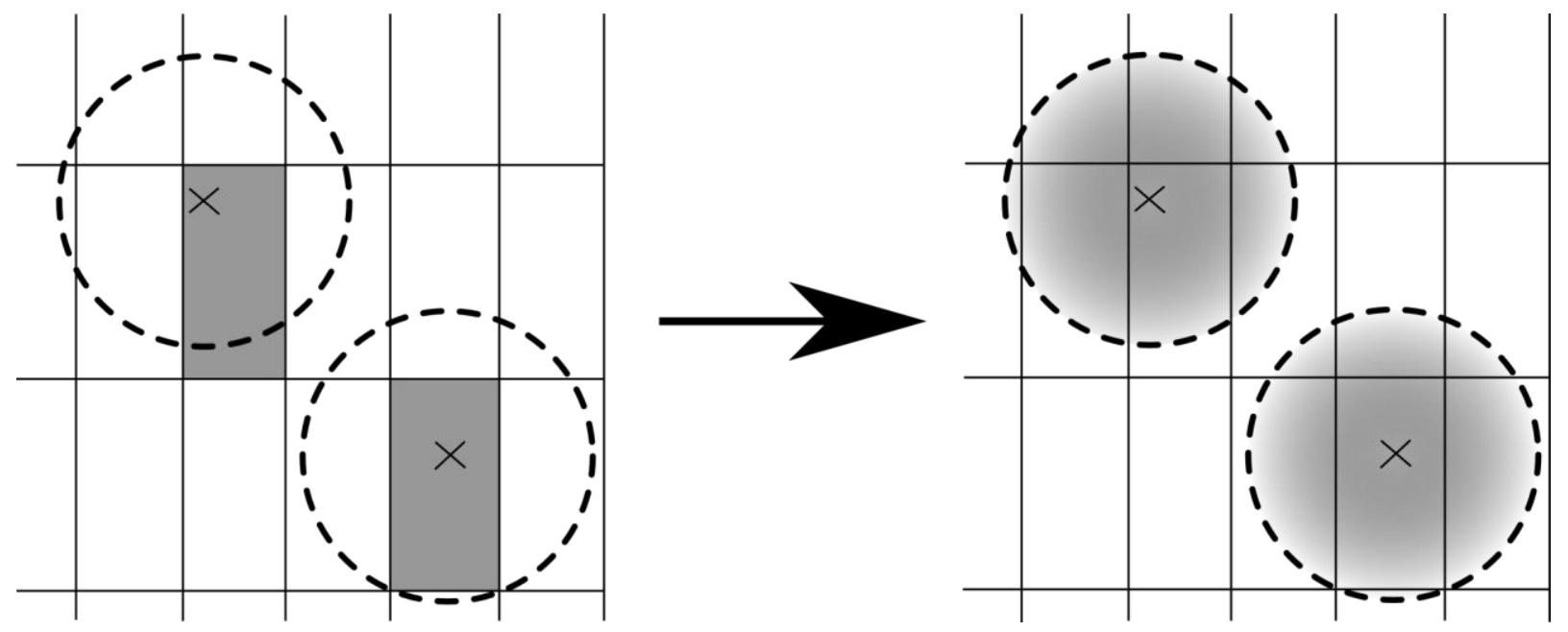

Fig. 2 : Scheme of the volume distribution process.

The void fraction in each cell is defined as the volume of gas divided by the volume of the cell. The volume distribution algorithm limits the void fraction values to a maximum of 1 and divides the reaction force between the cells, which is necessary for sake of grid size independency (see §4.4). This procedure is particularly essential in the present case of narrow channels and in the walls vicinity where the grid is preferentially refined.

\subsection{2 - Parallelization}

The DNS code has been parallelized according to the 2D decomposition approach in which the computational domain is partitioned in two directions only to keep a complete view in the working direction. For the bubbles equation, the computational domain is partitioned according to the $\mathrm{X}$ configuration (decomposition in $y$ and $z$ directions) and this configuration is maintained during all the resolution in order to simplify the boundary treatment at the subdomain interfaces. Each process handles its own set of bubbles, i.e. the bubbles located in its subdomain. When a bubble moves to another subdomain, it is removed from its initial process and added to the set of bubbles of its new process. This bubble data transfer between the two processes is performed through calls to the MPI library. 


\subsection{3 - Collisions sequence}

In the collision strategy proposed by Hoomans et al. (1996), the search for bubble-bubble or bubble-wall collisions requires the definition of two different time steps: a main constant time step used for time advancement of the Navier-Stokes equations, and a smaller variable time step used to displace the particles progressively in order to proceed to only one collision at a time.

The procedure set up in the present work is different: all bubbles are moved simultaneously after calculation of the continuous phase motion. The distance $\left\|\boldsymbol{D}_{i j}\right\|$ between two bubbles is calculated and collision occurs if this distance is smaller than the defined collision distance $D_{c o l}=r_{i}+r_{j}+h_{l}$ equal to the sum of bubbles radii and the lubrication distance $h_{l}=0.05\left(r_{i}+r_{j}\right)$. The position of interacting bubbles is corrected afterward considering that the collision occurred at a time $t_{c o l}$ comprised between the beginning and the end of the timestep. This strategy does not require substeps for the advancement of the dispersed phase but necessitates small displacements of the dispersed phase to avoid bubbles crossing each other. This hypothesis is justified by the use of relatively small time-steps. Indeed, the order of magnitude of bubble displacement can be estimated by $\Delta X^{*}=V^{*} \Delta t^{*}$, where the bubble velocity scale is $V^{*} \sim 1$. Hence, time-steps comprised between 0.001 and 0.01 should be sufficient to insure displacements smaller than bubbles radii, given that $r^{*} \sim 0.01-0.033$ in this work. Fig. 3 represents schematically the collision process between two bubbles.

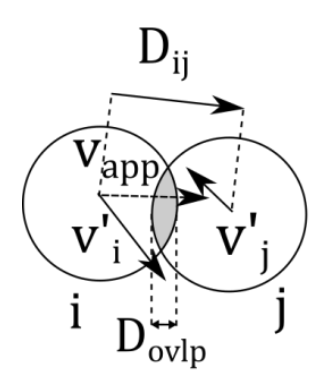

$(\mathrm{t}+\mathrm{dt})^{\prime}$
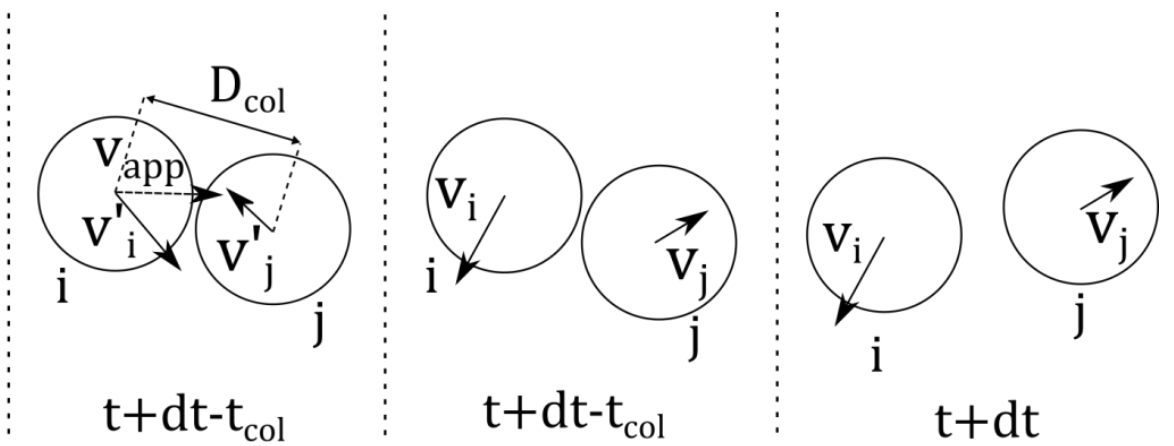

Fig. 3 : Scheme of a collision sequence. Collisions are not simulated at the time they occur, instead, bubbles positions and velocities are corrected once they already overlap the defined collision distance.

At time $(t+\Delta t)^{\prime}$, the approximated positions $\boldsymbol{x}_{i}{ }^{\prime}$ and $\boldsymbol{x}_{j}{ }^{\prime}$, and velocities $\boldsymbol{v}_{i}{ }^{\prime}$ and $\boldsymbol{v}_{j}{ }^{\prime}$ are calculated respectively for bubble $\mathrm{i}$ and $\mathrm{j}$ based on hydrodynamic interactions only. The vector 
that links the centers of mass of the two bubbles is $\boldsymbol{D}_{i j}$, the approach velocity is $\boldsymbol{v}_{a p p}=$ $\left(\boldsymbol{v}_{i}{ }^{\prime} \cdot \boldsymbol{D}_{i j}-\boldsymbol{v}_{j}{ }^{\prime} \cdot \boldsymbol{D}_{i j}\right) \frac{\boldsymbol{D}_{i j}}{\left\|\boldsymbol{D}_{i j}\right\|}$ and the overlap is computed from $D_{\text {ovlp }}=D_{\text {col }}-\left\|\boldsymbol{D}_{i j}\right\|$. The collision occurs actually at $t+\Delta t-t_{c o l}$, where $t_{c o l}=\frac{D_{o v l p}}{\left\|v_{a p p}\right\|^{\prime}}$, and the post-collision velocities are $\boldsymbol{v}_{i}=\boldsymbol{v}_{i}{ }^{\prime}-\left(1+\varepsilon_{r}\right) \frac{\boldsymbol{v}_{a p p}}{2}$ and $\boldsymbol{v}_{j}=\boldsymbol{v}_{\boldsymbol{j}}{ }^{\prime}+\left(1+\varepsilon_{r}\right) \frac{\boldsymbol{v}_{a p p}}{2}$, where $\varepsilon_{r}$ is the restitution coefficient. Thus, the actualized positions at $t+\Delta t$ are $\boldsymbol{x}_{i}=\boldsymbol{x}_{i}{ }^{\prime}-\left(1+\varepsilon_{r}\right) t_{c o l} \frac{\boldsymbol{v}_{a p p}}{2}$ and $\boldsymbol{x}_{j}=\boldsymbol{x}_{j}{ }^{\prime}+(1+$ $\left.\varepsilon_{r}\right) t_{c o l} \frac{v_{a p p}}{2}$.

Computational runs show a good agreement between results calculated with $\Delta t^{*}=0.01$ and $\Delta t^{*}=0.001$ for approximately 12,000 bubbles of size $r^{*}=0.03$. The averaged velocity profiles are almost identical while the averaged bubbles number and the averaged void fraction differences are of $3 \%$ only. The mean bubble displacements were also calculated and are respectively $\Delta X^{*}=0.007$ and $\Delta X^{*}=0.0007$, significantly lower than bubble size. Finally, the computation underdone with $\Delta t^{*}=0.01$ was almost 9 times faster for the same configuration. 


\section{3 - Coupling methodology}

\subsection{1 - Four-way coupling algorithm}

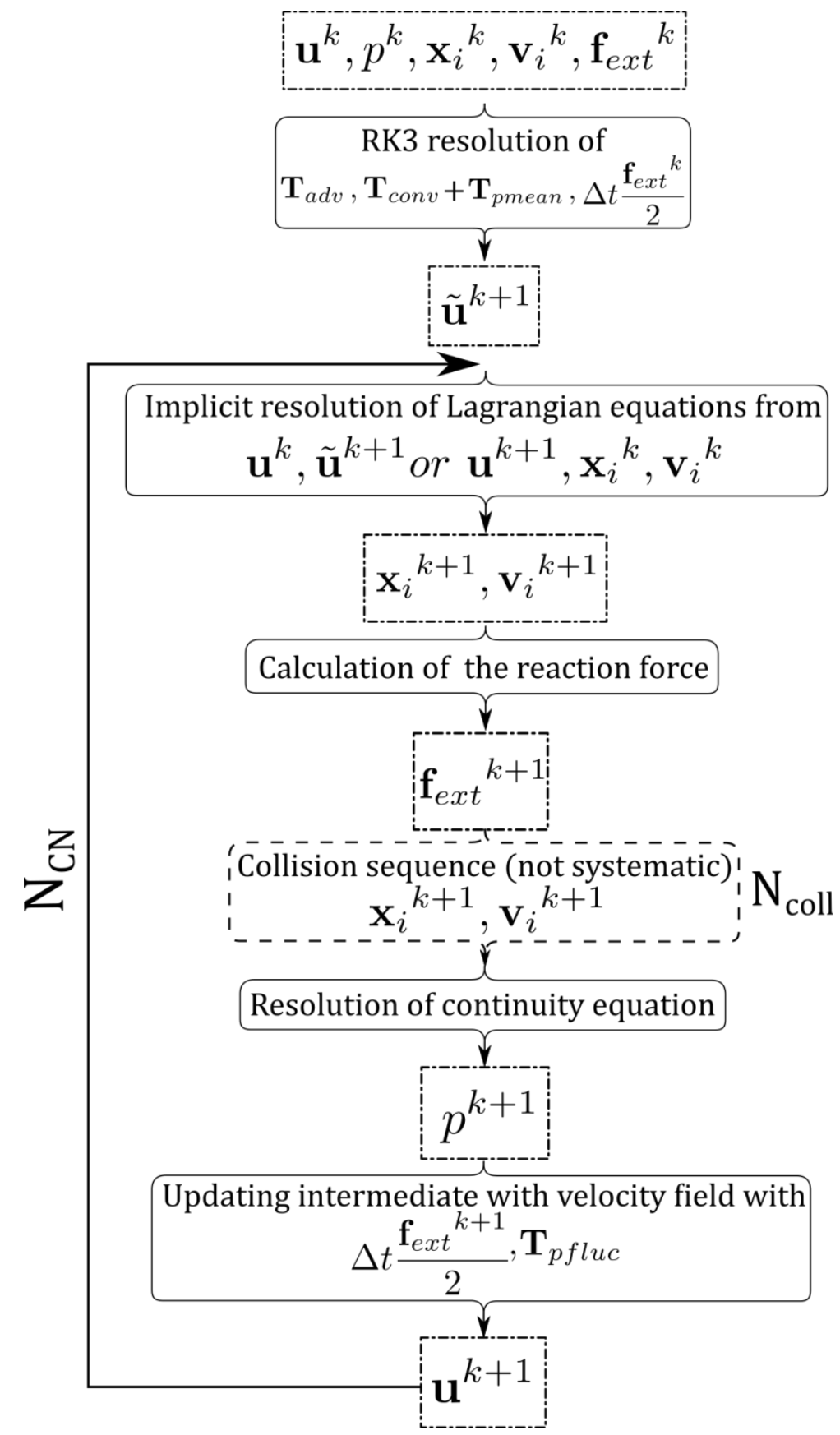

Fig. 4: Scheme of the numerical sequence for time advancement

Fig. 4 details the numerical procedure for the time advancement procedure with the coupling strategy. At time $k$, the velocity, pressure and external force field of the continuous phase are known, and position and velocities of each bubble as well. First, an intermediate 
velocity $\widetilde{\boldsymbol{u}}^{k+1}$ is calculated by explicit resolution of the advection and diffusion terms (Eqs. (12)) by the Runge-Kutta scheme (RK3) and the Euler evaluation of the mean pressure term. The force exerted by the bubbles is determined implicitly by the method of Cranck-Nicholson and half of the force field calculated based on time step $k$ is added to the intermediate velocity, at this stage.

The equation of motion is then resolved for each bubble based on the liquid velocities, $\boldsymbol{u}^{k}$ and $\widetilde{\boldsymbol{u}}^{k+1}$, estimated at the center of the spheres by a trilinear interpolation method. The calculation of the velocities $\boldsymbol{v}_{i}{ }^{k+1}$ is done by a Cranck-Nicholson implicit scheme. The external force field, applied by the dispersed phase on the continuous phase, is now calculated based on the new position and velocities. Each dispersed element applies a reaction force on the liquid which can be expressed by a combination of Eqs. (3) and Erreur ! Source du renvoi introuvable.: $\boldsymbol{F}_{D-C_{i}}{ }^{k+1}=-\boldsymbol{F}_{C-D, b_{i}}{ }^{k+1}=-\left(\left.\rho_{D} \frac{d v}{d t}\right|_{i} ^{k+1 / 2}+\left(\rho_{C}-\rho_{D}\right) \boldsymbol{g}-\left.\rho_{C} \frac{D \boldsymbol{u}}{D t}\right|^{k+1 / 2}\right)$.

Once all bubbles are moved, the bubble collision sequence is operated. This step could be very expensive in CPU time, if not prohibitive, in simulations of high bubbles number. In order to spare computing resources, one can decide not to compute the collision sequence at every Cranck-Nicholson iteration (see §3.3.2). In that case, bubbles are displaced without interacting with each other in the first Cranck-Nicholson iterations and collisions are computed in the last $N_{\text {coll }}$ iterations.

The last step is to resolve the continuity equation in order to calculate the fluctuating pressure terms and to update the final velocity $\boldsymbol{u}^{k+1}$. The procedure is iterated $N_{C N}$ times in order to calculate bubbles motion, i.e. the coupling force, based on $\boldsymbol{u}^{k+1}$ instead of $\widetilde{\boldsymbol{u}}^{k+1}$.

\subsection{2 $-N_{C N}$ and $N_{\text {coll }}$}

As stated before, two important iteration numbers can be tuned in the simulation: $N_{C N}$ and $N_{c o l}$. The sensitivity to these numbers is studied in the range $2 \leq N_{C N} \leq 10$ and $1 \leq N_{c o l} \leq N_{C N}$, considering the reference case to be $\left(N_{C N}=10, N_{c o l}=10\right)$. A very good precision is obtained for $N_{c o l} \geq 2$, regardless of $N_{C N}$. The CPU time for simulation parameters $\left(N_{C N}=2, N_{c o l}=2\right)$ and $\left(N_{C N}=2, N_{c o l}=1\right)$ is respectively 6 and 10 times smaller than for the 
reference case because of the collision sequence numerical cost for high bubbles number. The configuration for the following of this work is set to $\left(N_{C N}=2, N_{c o l}=2\right)$.

\section{4- Two-phase flow simulations}

\section{1 - One-way coupling approach with homogeneous modeling of the collective effects}

One major difficulty of multiphase flows modeling resides in describing the dispersed phase motion. Many strategies, going from the use of averaged empirical laws to direct simulation of the interfacial momentum exchange, were developed to this end. We propose to study here the Lagrangian tracking of a single bubble inside a virtual plume with an empirical modeling of the collective effects. A 2D-mapping of the void fraction is derived from the simulations of one gas-evolving electrode by the mixture model (Schillings et al., 2015) and applied to the DNS as a scalar function depending on both $x$ and $y$ directions. A Poiseuille flow is imposed at the entrance of the channel and the motion of a single bubble is calculated by means of Eq. (3). The collective effects due to bubble-bubble collisions are modeled by evaluating a drift velocity in the wall normal direction:

$$
v_{\text {drift }}=-r v_{\text {Stokes }} f(\alpha) D \vec{\nabla} \alpha-r^{2}|\dot{\gamma}| \beta(\alpha) \vec{\nabla} \alpha-r^{2}|\dot{\gamma}| \frac{\omega(\alpha)}{\tau} \vec{\nabla} \tau
$$

where $v_{\text {Stokes }}=\frac{2 g r^{2}}{9 v_{C}}$ is the settling velocity of a sphere in a quiescent liquid, $f(\alpha)=(1-\alpha)^{5}$ is a hindering function depending on the void fraction, $D$ is the hydrodynamic diffusion coefficient ( $\sim 1$ in the wall-normal direction), $\dot{\gamma}=\frac{\partial u_{x}}{\partial y}$ and $\tau=\mu_{C} \frac{\partial u_{x}}{\partial y}$ are the main shear-rate and shearstress, and $\beta(\alpha)=\frac{1}{3} \alpha^{2}\left(1+0.5 e^{8.8 \alpha}\right)$ and $\omega(\alpha)=0.6 \alpha^{2}$ are non-dimensional empirical coefficients depending on the void fraction.

Eq. (15) is based on the relative flux as defined in the previous work of Schillings et al. (2015) and is composed of the hydrodynamic self-diffusion, the shear-induced diffusion and the shear-induced migration. Hence, the drift velocity is a function of the continuous phase flow 
solved through DNS and the void fraction map obtained from the homogeneous model. The approximated velocity is thus $v_{y}+v_{\text {drift }}$ in $\boldsymbol{y}$ direction. The bubble is generated at the lowest point of the electrode and is then supposedly representative of the external bubble population of the plume. The bubble trajectory is thus compared to the evolution of the bubble plume thickness $\delta_{\alpha}$ computed with the homogeneous model along the electrode height. $\delta_{\alpha}$ is defined as a boundary layer thickness, i.e. the distance from the wall at which the void fraction recovers asymptotically (in our case $1 \%$ of the maximum value) its bulk value.

The numerical parameters of the DNS are listed in Table 1.

Table 1 : Numerical set-up of the DNS code for the Lagrangian tracking in a virtual plume

\begin{tabular}{|c|c|c|c|cc|}
\hline$\Delta \boldsymbol{t}^{*}$ & $\boldsymbol{N}_{\boldsymbol{x}} \times \boldsymbol{N}_{\boldsymbol{y}} \times \boldsymbol{N}_{\boldsymbol{z}}$ & $\boldsymbol{L}_{\boldsymbol{x}}^{*} \times \boldsymbol{L}_{\boldsymbol{y}}^{*} \times \boldsymbol{L}_{z}^{*}$ & $\boldsymbol{r}^{*}$ & \multicolumn{2}{|c|}{$\boldsymbol{R} \boldsymbol{e}_{\boldsymbol{h}}$} \\
\hline 0.001 & $83 \times 65 \times 33$ & $40 \times 2 \times 1$ & 0.025 & 50, & 108,200 \\
\hline
\end{tabular}

Fig. 5 shows the evolutions of plume thickness and bubble position in a channel with one gas-evolving electrode for different values of bubble production rates and the continuous phase Reynolds number. Symbols represent plume thicknesses obtained from the mixture model while the lines describe the outer bubble trajectories computed by DNS. Three cases are represented: $i=62 \mathrm{~A} \cdot \mathrm{m}^{-2}, R e_{h}=108$ (dash line \& triangles), $i=250 \mathrm{~A} \cdot \mathrm{m}^{-2}, R e_{h}=50$ (dot line \& circles), and $i=250 \mathrm{~A} \cdot \mathrm{m}^{-2}, R e_{h}=200$ (dash-dot line \& squares). The main objective of this study is to determine the sensitivity of the bubble plume thickness to the flow parameters. It can be observed in Fig. 5 that the bubble trajectory (DNS) is consistent with the boundary layer thickness evolution (homogeneous model). Both decrease with the Reynolds number in agreement with Schillings et al. (2015). 


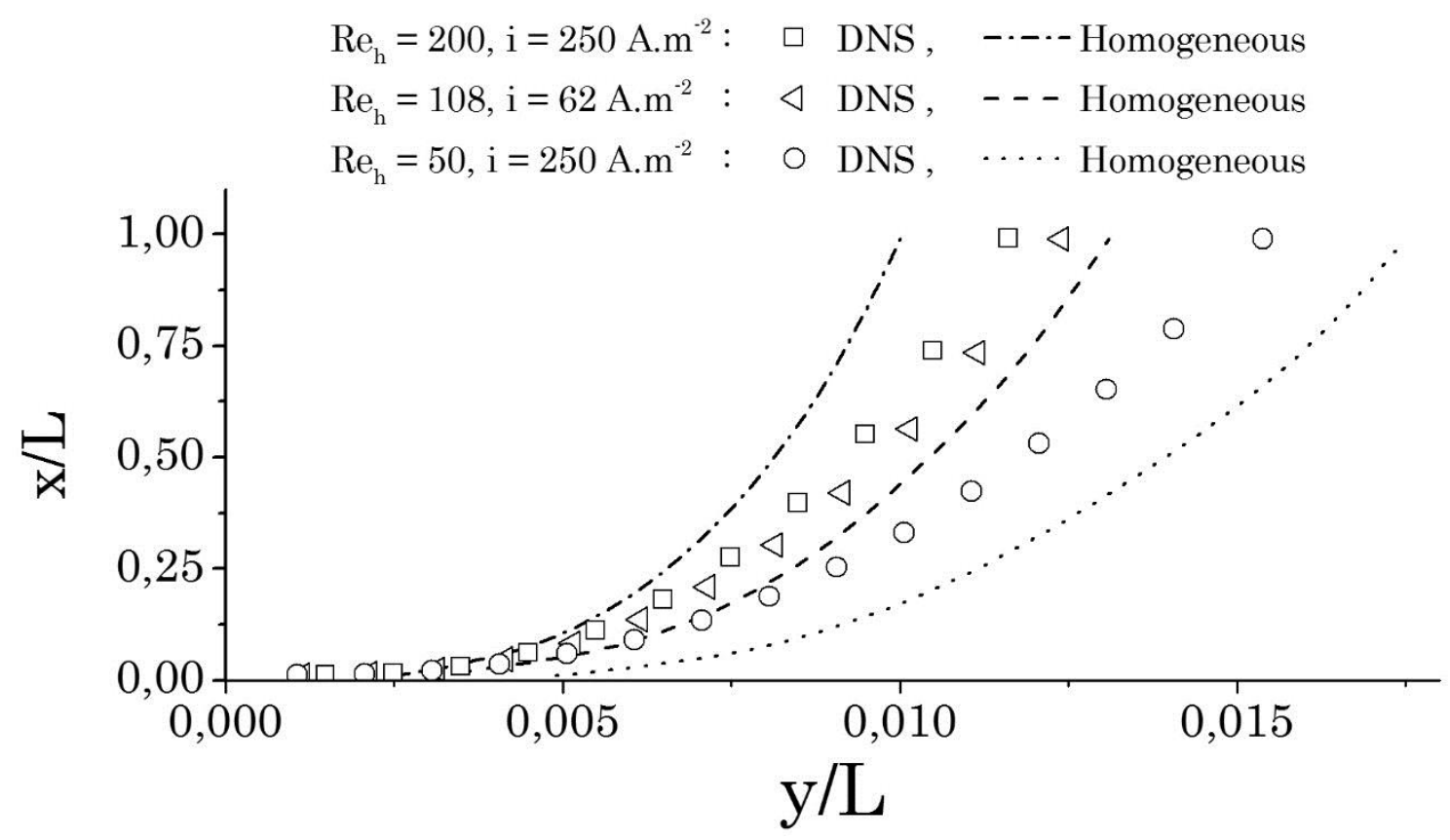

Fig. 5 : Comparison of bubble trajectory and plume thickness, at different values of bubble current density and Reynolds number. The squares, circles, cross and triangles correspond to boundary layer thickness, and the different lines are bubble trajectories. Distances are exceptionally scaled with the electrode length $\mathrm{L}$. $i=62 A$. $m^{-2}, R e_{h}=108$ (dash line \& triangles), $i=250 \mathrm{~A} \cdot \mathrm{m}^{-2}, R e_{h}=50$ (dot line \& circles), and $i=250 \mathrm{~A}$. $m^{-2}, R e_{h}=200$ (dash - dot line \& squares).

This hybrid approach using DNS to resolve the base flow and a closure term to model collective effects based on a void fraction is presented as a first application. It shows that the dispersion of bubbles can be evaluated qualitatively without taking into account collisions and that the general trend is respected between the DNS and the homogeneous model. This approach is still unideal because of its lack of genericity due to the empirical closure term and because it does not provide predictive results. Hence, complete resolutions of two-phase flows through DNS of the Eulerian-Lagrangian model and the wall-particles and inter-particles interactions are presented hereafter.

\section{2 - Four-way coupled simulation of a practical case: the vertical electrolysis cell}

Results obtained by a DNS of the two-phase flow evolving between vertical plates are presented in this section. The former are confronted to the homogeneous model (Schillings et al., 2015) and experiments (Boissonneau and Byrne, 2000). The reference case is a water electrolysis operated at $2000 \mathrm{~A} / \mathrm{m}^{2}$. 
The simulation parameters are given in Table 2. The number of bubble generated at each $\Delta t^{*}, \dot{N b}$, is 2 times larger at the hydrogen $\left(y^{*}=0\right)$ than at the oxygen $\left(y^{*}=2\right)$ production sides, due to the nature of the electrochemical reactions.

Table 2 : Numerical set-up of the vertical water electrolysis simulation, based on (Boissonneau and Byrne, 2000).

\begin{tabular}{|c|c|c|c|c|c|c|}
\hline$\Delta t^{*}$ & $N_{x} \times N_{y} \times N_{z}$ & $L_{x}^{*} \times L_{y}^{*} \times L_{z}^{*}$ & $\boldsymbol{r}^{*}$ & $\boldsymbol{R} e_{\boldsymbol{h}}$ & $\boldsymbol{N} b_{y^{*}=0}$ & $\mathbf{N b}_{y^{*}=2}$ \\
\hline 0.01 & $65 \times 33 \times 17$ & $26.7 \times 2 \times 0.93$ & 0.023 & 108 & 1 & 0.5 \\
\hline
\end{tabular}

\subsection{1 - Instantaneous flow}

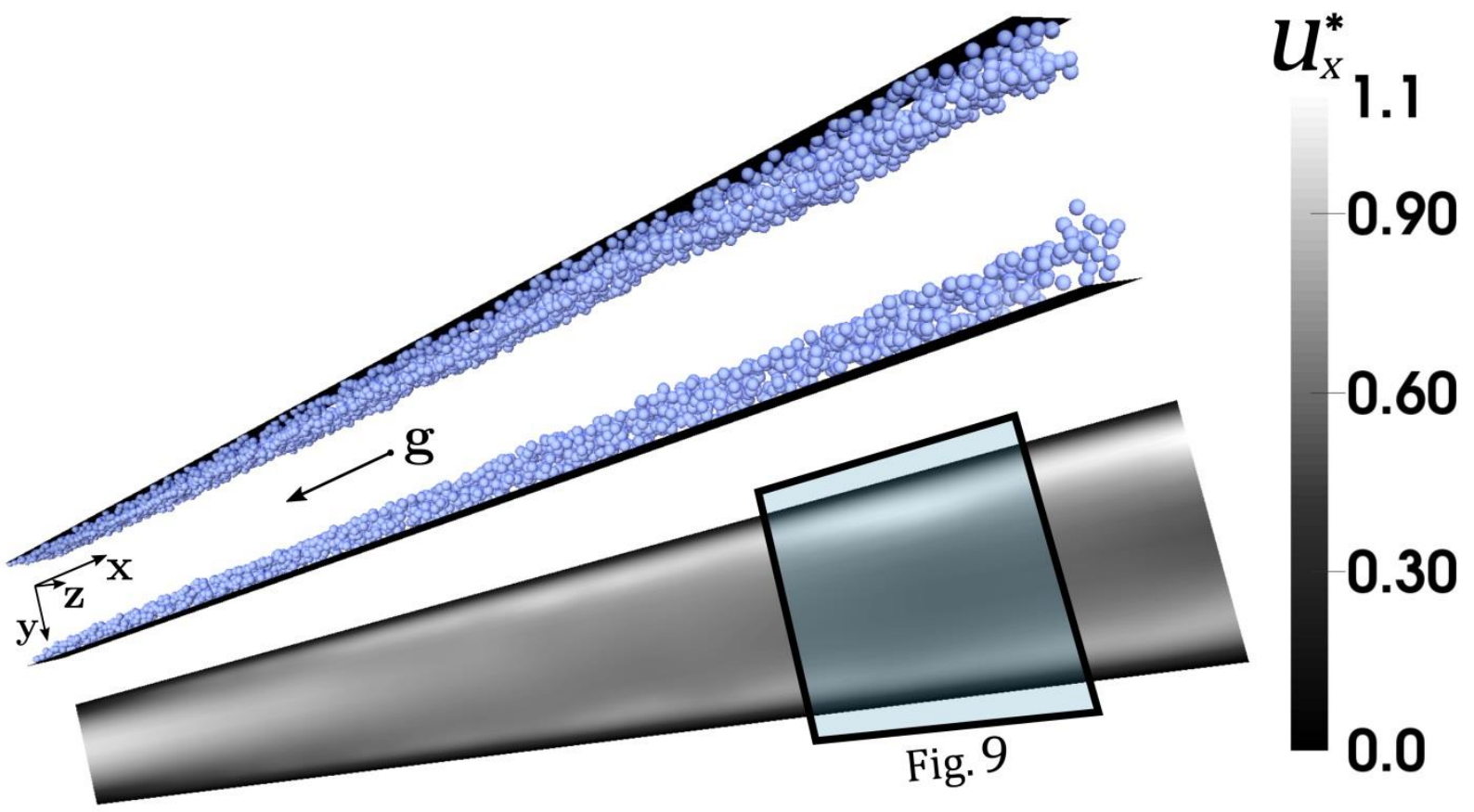

Fig. 6 : Representation of the DNS results at time $t^{*}=80$. On top is a view of electrodes and bubbles for a sample of the domain (approx. one third in $z$ direction). The grayscale surface represents the instantaneous streamwise velocity averaged along $z$.

Fig. 6 is a snapshot of the flow state and bubble organization at time $t^{*}=80$ (corresponding to 1s) for which the averaged statistics of the two-phase flow are stationary. The sample is shown on the whole $\boldsymbol{x}$ and $\boldsymbol{y}$ directions while its depth is 0.35 along $\boldsymbol{z}$. A 3D view of the electrodes and the bubble plume can be seen at the top of Fig. 6. The corresponding 
spanwise-averaged velocity of the continuous phase is presented at the bottom of Fig. 6 . Velocity profiles are presented in Fig. 7 for three sections of the channel.

The reader might refer to the video (link) for a 3D time evolving visualization of the computed flow.
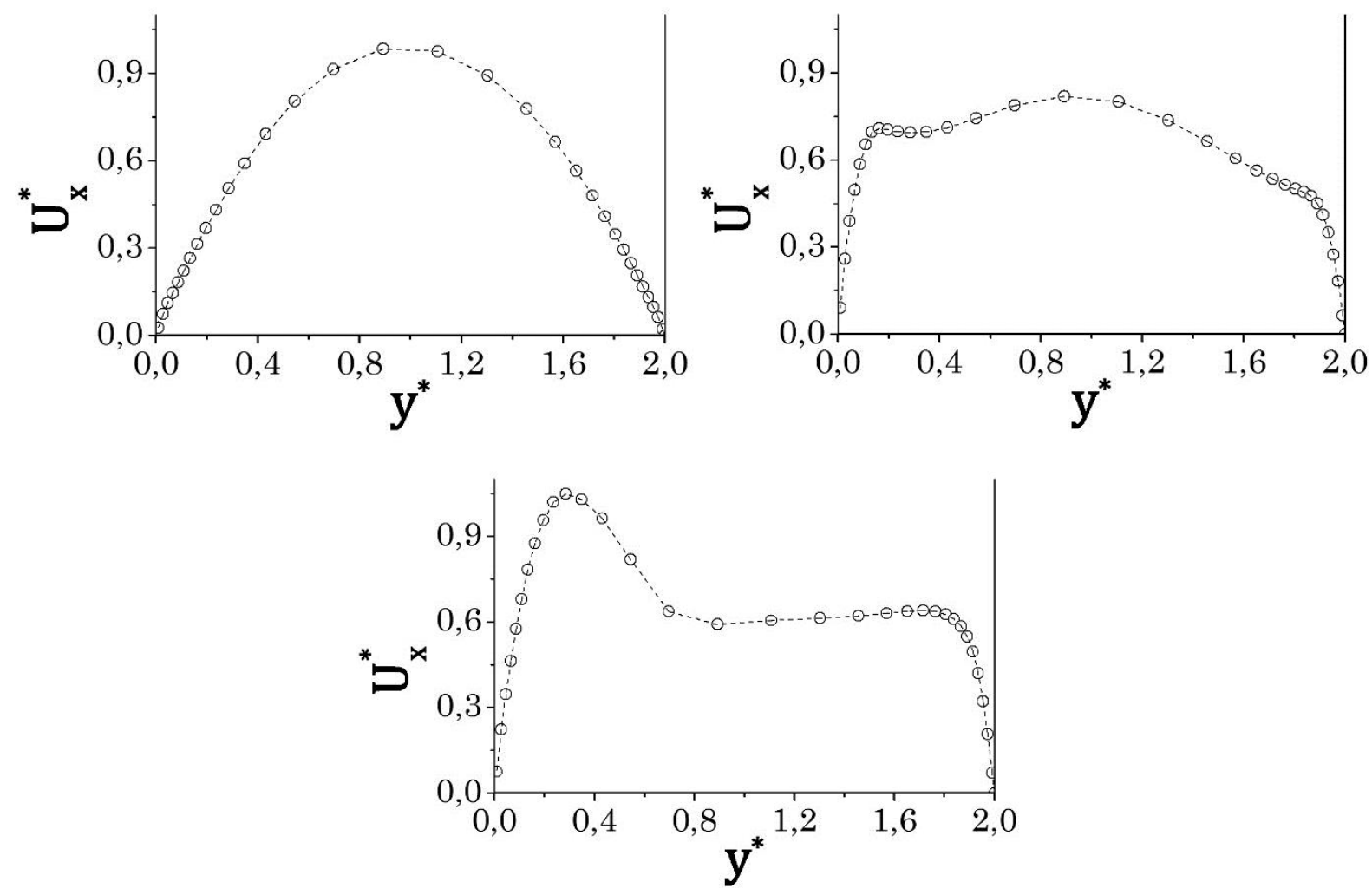

Fig. 7 : Evolution of the velocity field at three channel positions. (a) top-left: inlet, (b) top-right: half-height, (c) bottom: outlet

The flow enters the channel at the inlet (Fig. 7a) with a standard Poiseuille profile. It accelerates progressively near the walls (Fig. 7b) due to the buoyancy forces until reaching a maximum velocity at the outlet (Fig. 7c) of $u_{x}=1.0$ on the hydrogen side $\left(y^{*} \approx 0.3\right)$ and $u_{x}=0.64$ on the oxygen side $\left(y^{*} \approx 1.8\right)$. The dissymmetry of the profiles is caused by the differences in bubbles production rates. Since the flow rate is conserved in the computational domain, the velocity decreases at the center until a minimum of $u_{x}=0.60$ at the end of the electrode. 
Slight velocity oscillations along the $y$-axis are observed in the 3D time-evolving visualizations and they are found to coincide with the apparition of wavelets in the bubble plumes. This phenomenon can be explained by the non-uniformity of the collision effects due to local variations of bubbles population. As a consequence, the bubble curtain thickness cannot grow homogeneously along the $x$-direction and experiences small spatio-temporal oscillations.

Slight time fluctuations are for instance seen in Fig. 8 that shows the total number of bubbles contained in the computational box versus the non-dimensional time.

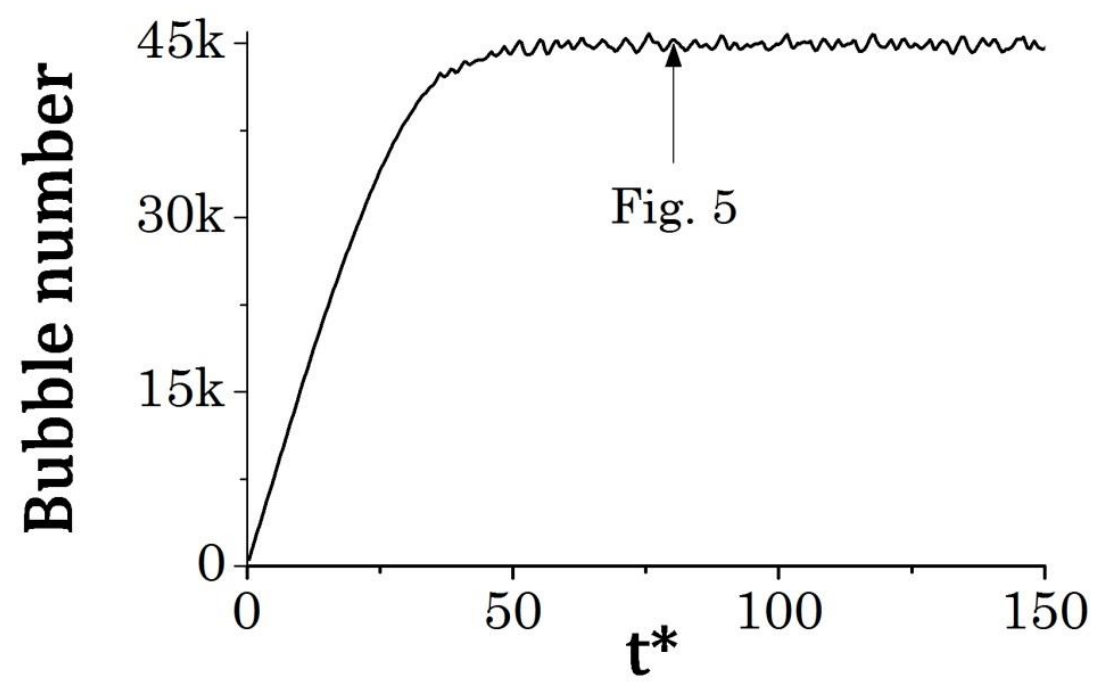

Fig. 8 : Evolution of the bubble number versus the non-dimensional time.

From Fig. 8 it can be seen that the number of bubble increases until reaching a stationary state at $t_{\text {stat }}^{*}=40$. The flow is considered steady beyond that point and the number of bubbles oscillates slightly around 44.884 bubbles with a maximum variation of about $+-2 \%$. The data is space and time averaged in $z$ direction for a period $T_{\text {stat }}^{*}$, starting from $t_{\text {stat }}^{*}+10$ and sampling every $\Delta t_{\text {stat }}^{*}$. Here the minimum values are set to $T_{\text {stat }}^{*}=50$ and $\Delta t_{\text {stat }}^{*}=2$ but it can vary depending on the oscillations. The Root Mean Square (RMS) deviation is also calculated in order to quantify temporal variations of the flow fields, i.e. the effects of the inclusions on the 
continuous phase. The notations for the average and RMS of a variable are respectively $\overline{\operatorname{var}}^{t, z}=\frac{\sum_{t}\left[\frac{\sum_{z} v^{*} r^{*} \Delta z}{L_{z}}\right] \Delta t_{\text {stat }}}{T_{\text {stat }}}$ and $\operatorname{var}^{R M S}=\frac{\sum_{t}\left[\frac{\sum_{z}\left|\operatorname{var}^{*}-\overline{v_{z}} t, z\right| \Delta z}{L_{z}}\right] \Delta t_{\text {stat }}}{T_{\text {stat }}}$.

Fig. 9 shows a magnified view part of the channel, corresponding to the window region depicted in Fig. 6. The small dark points indicate each bubble center in the $(x, y)$ plane. Fig. 9a displays $\frac{f_{\text {ext }, x}}{\rho_{C} u_{C P} \frac{1}{h}}$ while Fig. 9b displays $u_{x}^{*}$. It is obvious that the force exerted by the bubbles is proportional to the bubble concentration. However the maximum velocity is found at an intermediate location (between the wall and the core flow) in a way similar to classical free convection.
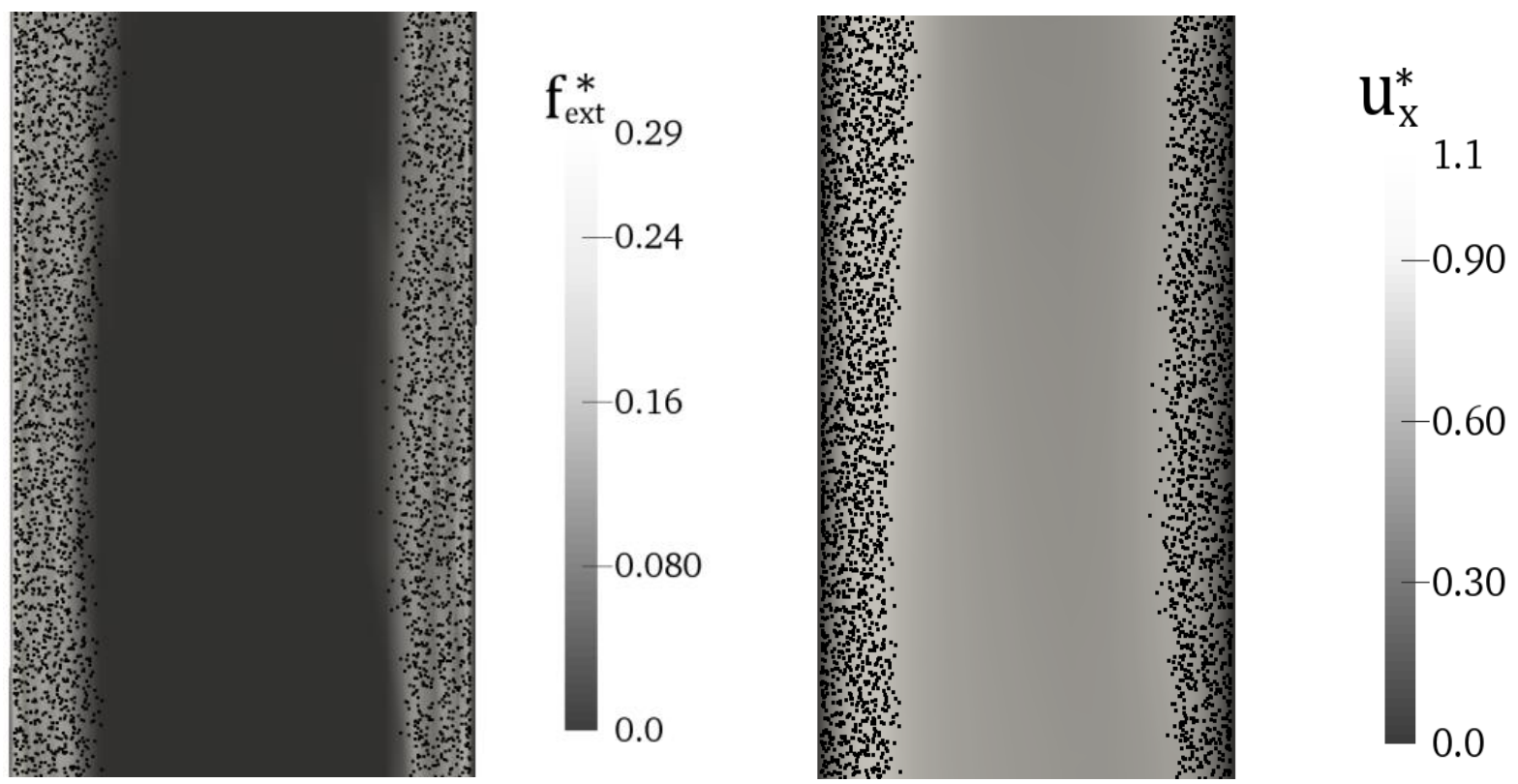

Fig. 9 : Front visualization of the channel at the height defined by the window drawn of Fig. 6 . The centers of bubbles are represented by points. The grayscale displays (a) left : the non-dimensional force exerted on the liquid by the bubbles and (b) right : the non-dimensional streamwise liquid velocity.

\subsection{2 - Time-averaged profiles}

The streamwise velocity profiles obtained by DNS at the outlet of the electrodes are compared to the Laser Doppler Velocimetry measurements of Boissonneau and Byrne (2000) and to the 2D homogeneous model simulation of Schillings et al. (2015) in Fig. 10. The inlet Poiseuille profile common to the three cases is also depicted in complement. 


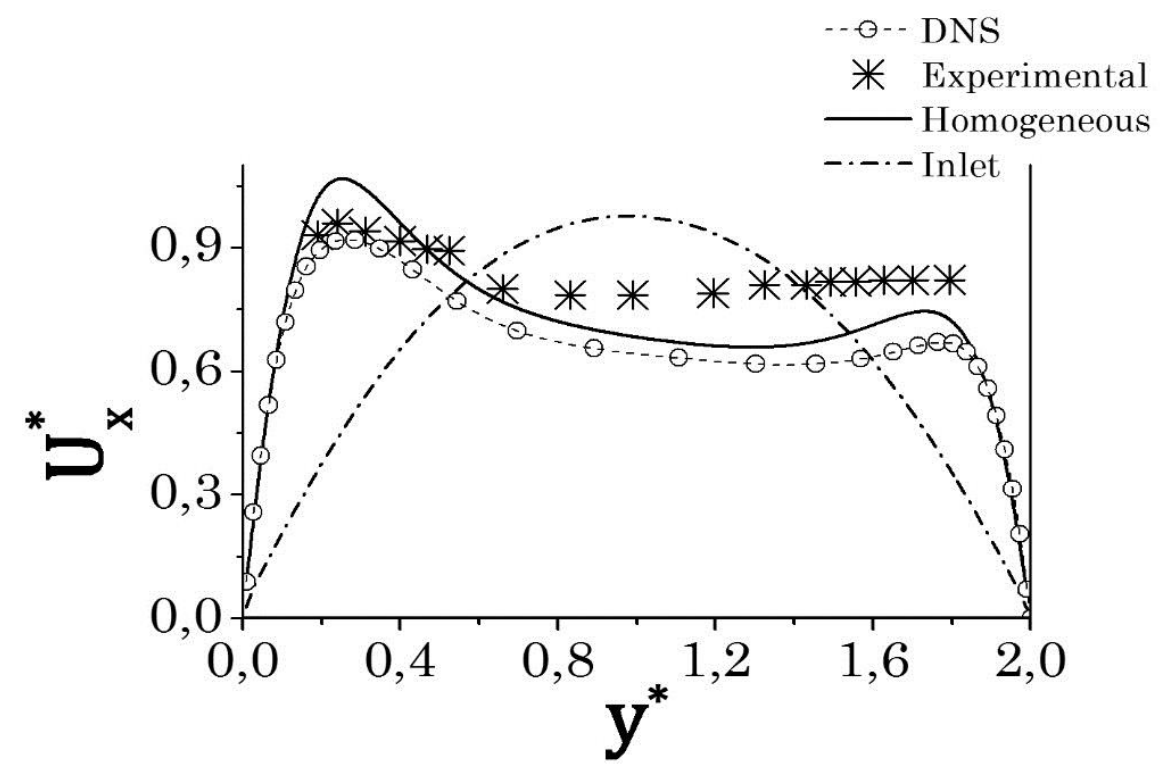

Fig. 10: Experimentally measured and simulated liquid velocity profiles. Measurements (crosses), stationary simulation from the homogeneous model (full line) and time- and z-averaged data from DNS (circles) are plotted at electrode outlet. The inlet Poiseuille profile is represented by the dash-dot line.

As stated by Schillings et al. (2015), the measurements performed by Boissonneau and Byrne (2000) do not respect the flow rate conservation. Hence, their results cannot be used directly as a reliable quantitative reference but rather as an indicator of the global behavior for the velocity field. Keeping that in mind, the agreement between simulations and experiments is satisfying. Surprisingly, both simulations give similar results despite the simple collision algorithm used in the DNS case at this stage. One will also note that those results were obtained in 7.5 hs by parallel computation on an Intel ${ }^{\circledR}$ Xeon E5520 (4 cores of $2.27 \mathrm{GHz}$ ), which is a very reasonable duration for a simulation comprising almost 45.000 bubbles.

Unfortunately, there is no experimental data combining both velocity and void fraction measurements in the literature in the case of an electrolysis cell or a similar process, to the best authors' knowledge. Consequently, the following results compare simultaneously the void fraction and velocity distributions stemming from DNS to the data from the mixture model only. 


\section{3 - Bubble dispersion}

\subsection{1 - Comparison with the mixture model}

The evolution of bubble curtains is compared between the mixture model simulations (Schillings et al., 2015) and the DNS. Two simulations of one gas-evolving electrode referred to as the high current density (HCD) and the low current density (LCD) cases are presented. There is actually no absolute "high" or "low" value of current densities because of many parameters (bubble size, liquid flow, electrode length and interspace) that enter in the two-phase flow development process. However, Schillings et al. (2015) noticed by means of their homogeneous model two characteristic void fraction evolutions, that we name the "flat" and "diffusive" shapes. The different terms involved in the relative void fraction flux (hydrodynamic diffusion, shear-induced diffusion and shear-induced migration) do not have the same magnitude depending on the two-phase flow conditions, and the resulting shape of the void fraction is linked the dominating term. The first case presented in Fig. 11 coincides principally with relatively high current densities and small bubbles and is representative of a dominating shearinduced migration term. The second case presented in Fig. 12 is generated at relatively low current densities with large bubbles and holds for a void fraction diffusion led by the hydrodynamic self-diffusion term. The numerical set-up of the DNS for both cases is presented in Table 3.

Table 3 : DNS parameters for the HCD case (second line) and the LCD case (third line)

\begin{tabular}{|l|c|c|c|c|c|c|}
\cline { 2 - 7 } \multicolumn{1}{c|}{} & $\Delta \boldsymbol{t}^{*}$ & $\boldsymbol{N}_{\boldsymbol{x}} \times \boldsymbol{N}_{\boldsymbol{y}} \times \boldsymbol{N}_{\boldsymbol{z}}$ & $\boldsymbol{L}_{\boldsymbol{x}}^{*} \times \boldsymbol{L}_{\boldsymbol{y}}^{*} \times \boldsymbol{L}_{\boldsymbol{z}}^{*}$ & $\boldsymbol{r}^{*}$ & $\boldsymbol{R e}_{\boldsymbol{h}}$ & $\boldsymbol{N} \boldsymbol{b}_{\boldsymbol{y}^{*}=\mathbf{0}}$ \\
\hline Fig. 11 & 0.01 & $65 \times 33 \times 17$ & $16 \times 2 \times 0.94$ & 0.03 & 100 & 10 \\
\hline Fig. 12 & 0.01 & $65 \times 33 \times 17$ & $5.4 \times 2 \times 6.3$ & 0.033 & 165 & 2.5 \\
\hline
\end{tabular}

Two different meshing strategies are also compared for both cases, with the same number of nodes. The "fine grid" is refined on the $y$ direction near the wall and the smallest cell size is $0.5 r$. The "coarse grid" has a constant size approximately equal to $1.5 r$. 

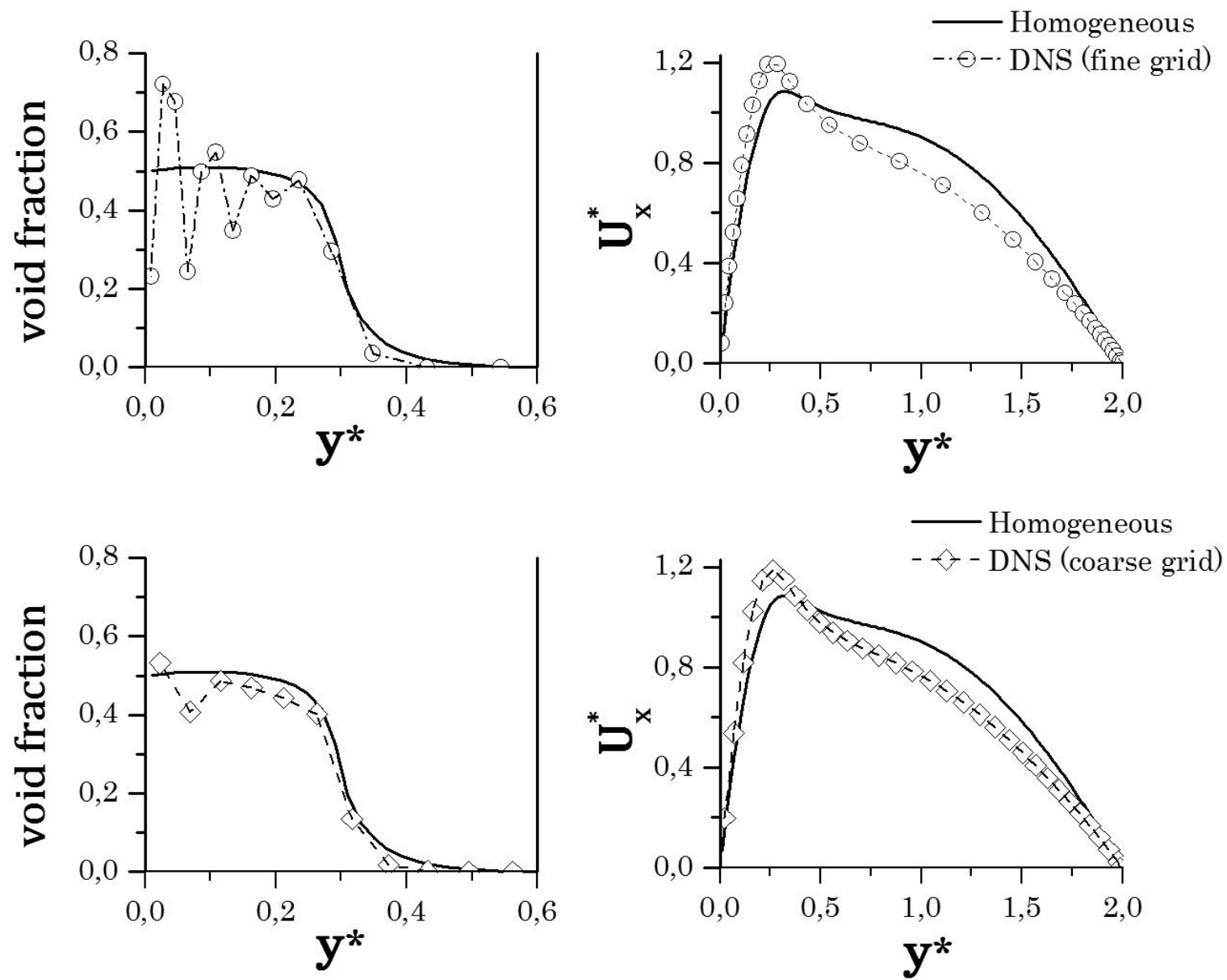

Fig. 11 : Simulations of the HCD gas-evolving electrode. Results from DNS computed with a fine grid (circles) are shown in the top figures and those computed with a coarse grid (diamonds) are in the bottom ones. They are compared to the same homogeneous model simulation (full line). (a) top-left : mean void fraction, fine grid; (b) top-right : mean streamwise velocity, fine grid; (c) bottom-left : mean void fraction, coarse grid; (d) bottom-right : mean streamwise velocity, coarse grid. Results are plotted at the electrode outlet. 

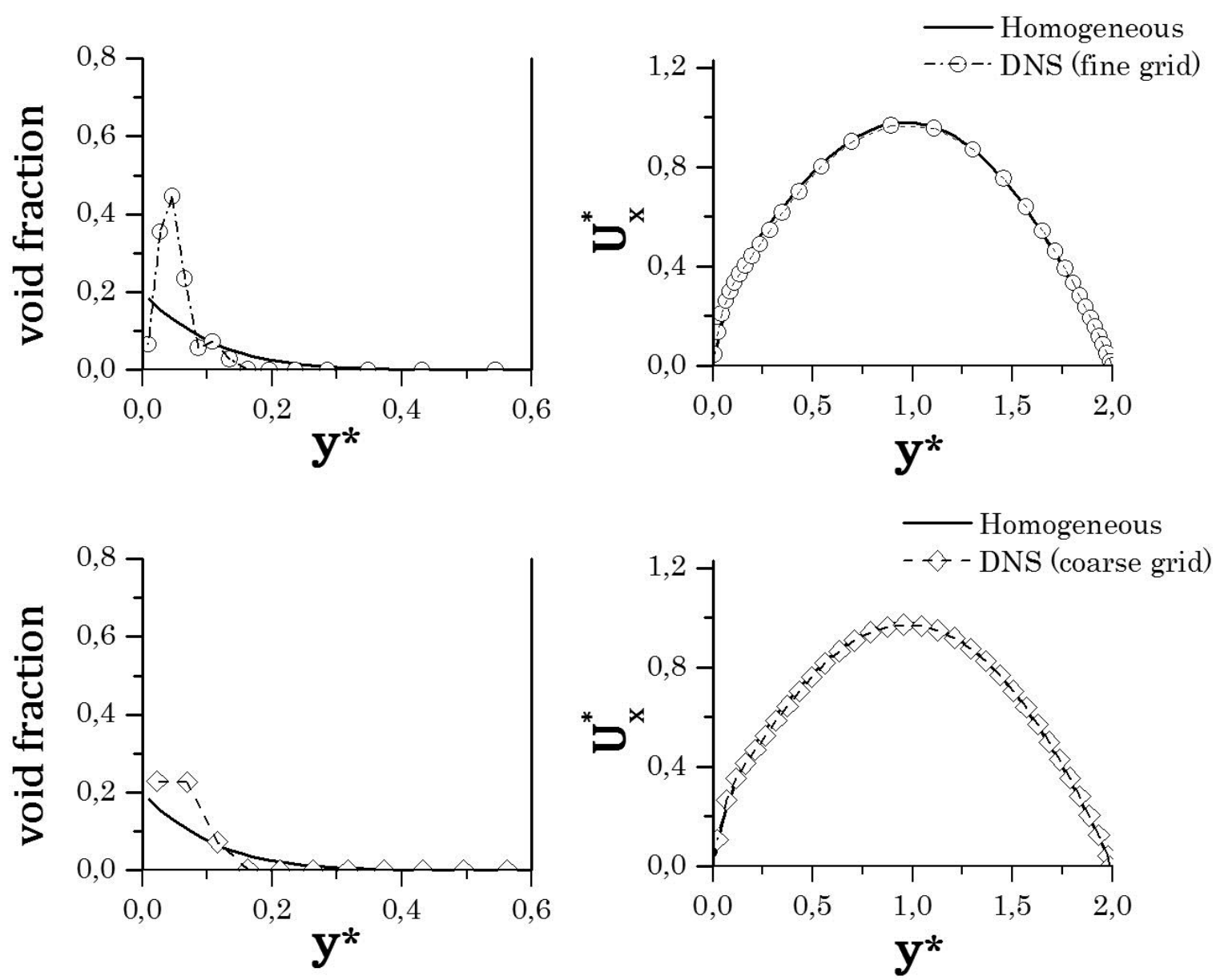

Fig. 12 : Simulations of the LCD gas-evolving electrode. Results from DNS computed with a fine grid (circles) are shown in the top figures and those computed with a coarse grid (diamonds) are in the bottom ones. They are compared to the same homogeneous model simulation (full line). (a) top-left : mean void fraction, fine grid; (b) top-right : mean streamwise velocity, fine grid; (c) bottom-left : mean void fraction, coarse grid; (d) bottom-right : mean streamwise velocity, coarse grid. Results are plotted at the electrode outlet.

Fig. 11 and Fig. 12 compare the void fraction and velocities computed by DNS to the homogeneous model simulations. In the HCD case (Fig. 11), there is a very good matching between the homogenous model simulation results and the DNS results, both in terms of the magnitude of the void fraction and the plume thickness (Fig. 11a). The common trend observed in the DNS and in the homogeneous model simulation is that the void fraction is elevated for $y^{*}<0.3$ and decreases sharply at the outer limit of the bubble curtain. We call that a "flat" profile. The noticeable dissimilarity though is the void fraction peaks values, which arise from the difference of bubbles representation between the homogeneous model and the Lagrangian formulation. In the former case the dispersed phase is seen as a scalar generated by a flux at the 
wall and transported in the continuous phase. The void fraction is maximum (or close to the maximum) at the wall and evolves smoothly away from it. In the latter case however, bubbles are spherical objects which implies a non-regular repartition of their volume. Because the attachment of bubbles to the wall during their growth is not modelled, the void fraction at $y=0$ is necessarily zero. Additionally, the hypothesis of not coalescing bubbles leads to a decrease of the void fraction in the interspace between them. The volume distribution procedure described in $\$ 3.2 .1$ allows the use of numerical cells smaller than the bubbles and thus provides an opportunity for visioning the non-regular repartition of bubble volumes, as seen in Fig. 11a. Those results are obtained with cells size of half the bubble radius near the wall and are also confirmed by finer meshes. The void fraction peaks situated at intervals of approximately one bubble diameter can be leveled by computing the DNS with a coarser grid (1.5r) as presented in Fig. 11c. This has the effect of averaging the void fraction which is then very close to the homogeneous profile.

The velocity profiles however are identical for both "fine grid" (Fig. 11b) and "coarse grid" (Fig. 11d) cases and thus validate the two different void fraction profiles. The acceleration of the liquid near the wall matches well with the homogeneous model. The wall velocity gradients are very similar and the maximum velocities calculated by the DNS and the homogeneous model show a difference of only $9 \%$.

The LCD case presents a higher discrepancy between the DNS and the homogeneous model simulation, as shown in Fig. 12. Although the matching in terms of velocity (Fig. $12 b, d)$ is excellent, the evolution of void fraction (Fig. 12a,c) is quite different. The void fraction computed by the homogeneous model is maximal at the wall and decreases progressively along $y$ axis. We call that a "diffusive" behavior due to the similarity with the temperature profile in thermal boundary layers. The maximum void fraction computed by the DNS with a fine grid (Fig. $12 \mathrm{a}$ ) is more than twice that of the homogeneous simulation and the plume thickness is almost 2 times smaller. The void fraction peak is leveled by use of the "coarse grid" (Fig. 12ac) and is much closer to the homogeneous model value. We do not clearly retrieve the diffusive behavior observed in the homogeneous model by DNS which is probably explained by the very different dispersed phase models. 
Globally, the results of our study are very encouraging in that they corroborate those computed in previous works (Schillings et al., 2015). Additionally, it is seen that the DNS allows a local description of the dispersed phase that is, by definition, impossible with the homogeneous model. As a consequence, the question arises as to whether the representation by the homogeneous model of bubble dispersion as a diffusive process in the LCD case is correct or not. The DNS reveals a dispersion process caused only by collisions and the observed void fraction peaks at regular intervals, corresponding to bubble diameters, seem to reflect a growth of the bubble plume in the form of piling layers. Comparison with reliable measurements of the void fraction and a close observation of the plumes will be necessary to confirm these results.

\subsection{2 - Effect of the restitution coefficient}

It is evident so far that the growth of plume thickness is driven by inter-particle collisions, but the results shown in Fig. 11 and Erreur! Source du renvoi introuvable. suggest that those collisions do not have a strong dispersive effect and lead instead to the superposition of bubbles layers. Recalling the assumption of inelastic collisions that hold for the considered bubbly flows, we propose to determine if the dissipation of collisions kinetic energy has a strong impact on the void fraction profile. A comparison of the reference case $\left(\varepsilon_{r}=0\right)$ to the opposite fully elastic collisions assumption $\left(\varepsilon_{r}=1\right)$ is presented hereafter. The set of parameters previously indicated for the HCD case is kept here (see Table 3) and the uniform "coarse grid" is used. Fig. 13 shows the void fraction profiles computed at the outlet of the electrode. Circles and stars correspond to $\varepsilon_{r}=0$ and $\varepsilon_{r}=1$ respectively. 


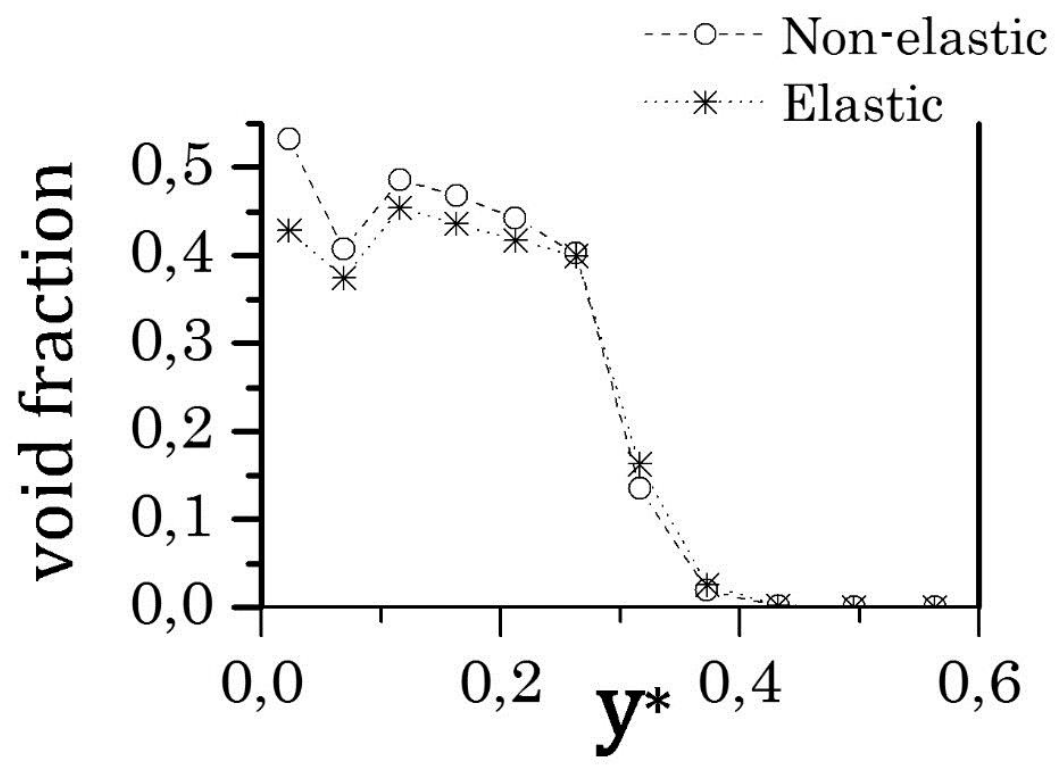

Fig. 13 : Mean void fraction in the HCD case with non-elastic (circles) and fully elastic (stars) collisions. Results are plotted at the electrode outlet.

Surprisingly, there is no major disparity in terms of dispersion along $y$ axis between the elastic and non-elastic collisions assumptions. This could be explained by the fact that collisions occur at low velocities in that direction and the observed dispersion is more representative of an obstruction phenomenon than on literally colliding spheres. Still, the void fraction magnitude is slightly lower when considering fully elastic collisions. Bubbles streamwise velocity is indeed largely higher than the wall normal velocity and elastic collisions along $x$ axis are probably much more intense. Elastic collisions would thus enhance bubble evacuation and lead to a slight reduction of the averaged void fraction.

\section{4 - Mesh sensitivity}

From a numerical point of view, the question of dependency to the grid size naturally arises and requires a detailed investigation. The point force approximation necessary to the Lagrangian description of the dispersed phase classically requires bubbles being much smaller than the numerical cells. However, this restriction should be overcome by means of the volume distribution algorithm discussed in §3.2.1. Sensitivity of $\bar{u}^{t, z}, \bar{\alpha}^{t, z}$ and $u^{R M S}$ to the mesh refinement in the $y$ and $z$ directions is studied for uniform grids in the range $43 \times 17 \times 13$ to $43 \times 65 \times 49$. The bubble size is chosen so that the bubble radius to cell size ratio varies from 
12 to 3 for the coarser and finer meshes, respectively. Because of the small bubble radius studied here and in order to visualize a substantial back force effect on the base flow with a limited population of inclusions, the gravitational acceleration is artificially set to $g=800 \mathrm{~m}$. $s^{-2}$. The global numerical parameters are listed in Table 4.

Table 4 : Numerical set-up of the mesh sensitivity study.

\begin{tabular}{|c|c|c|c|c|c|}
\hline$\Delta \boldsymbol{t}^{*}$ & $\boldsymbol{N}_{\boldsymbol{x}} \times \boldsymbol{N}_{\boldsymbol{y}} \times \boldsymbol{N}_{\boldsymbol{z}}$ & $\boldsymbol{L}_{\boldsymbol{x}}^{*} \times \boldsymbol{L}_{\boldsymbol{y}}^{*} \times \boldsymbol{L}_{z}^{*}$ & $\boldsymbol{r}^{*}$ & $\boldsymbol{R} \boldsymbol{e}_{\boldsymbol{h}}$ & $\boldsymbol{N} \boldsymbol{b}_{\boldsymbol{y}^{*}=\mathbf{0}}$ \\
\hline 0.001 & $43 \times 17 \times 13,43 \times 33 \times 25$, & $16 \times 2 \times 1.6$ & 0.01 & 100 & 0.5 \\
& $43 \times 49 \times 37$ and $43 \times 65 \times 49$ & & \\
\hline
\end{tabular}

The first important result of this study is that the averaged velocities and void fraction are not dependent on the mesh refinement. It confirms thus the efficiency of the bubble volume distribution strategy in extending the genericity of the point force approximation and suppressing the restriction on the bubble to mesh sizes ratio. The choice of a particular mesh is thus guided by two-phase flow considerations only. Refinement of the grid in the vicinity of the walls might turn out to be necessary, e.g. due to elevated velocity gradients induced by the buoyancy effects or in order to visualize the void fraction peaks (as evoked in §4.3.1).

The second aspect evaluated here concerns time depending statistics which are valuable because they traduce the bubble-induced fluctuations of the velocity field in a normally laminar flow. The RMS of the velocity is an interesting data in mixing problematics for instance and has applications by extension to the study of turbulence modulation. The profiles of $u_{x}^{R M S}, u_{y}^{R M S}$ and $u_{z}^{R M S}$ at the outlet of the electrodes are plotted in Fig. 14. 

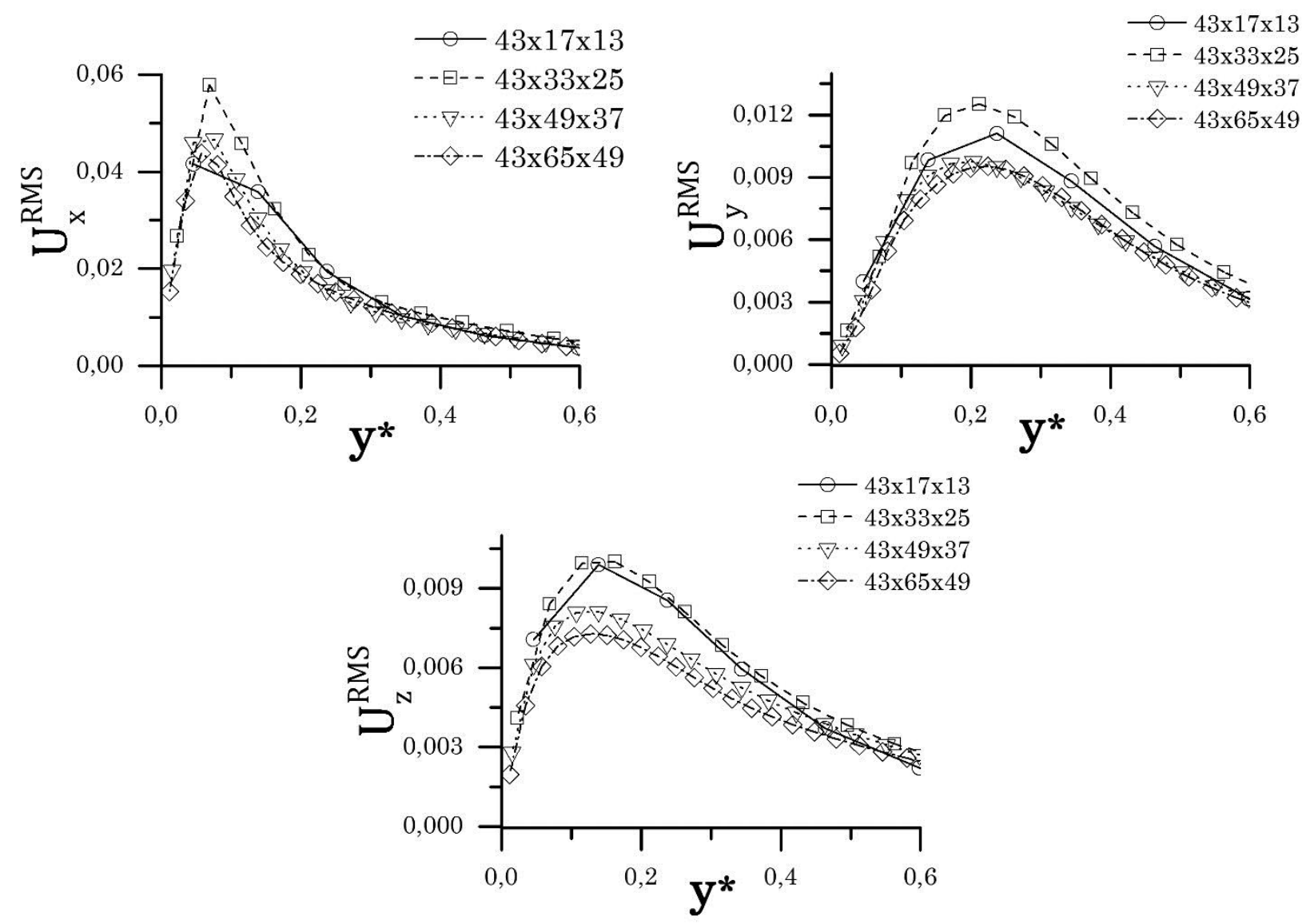

Fig. 14 : Mesh sensitivity of the RMS of velocity components versus y-axis, at the outlet of the electrodes. (a) topleft : $u_{x}^{R M S}$, (b) top-right : $u_{y}^{R M S}$, (c) bottom : $u_{z}^{R M S}$. Computations are run using four different meshes: (circles)

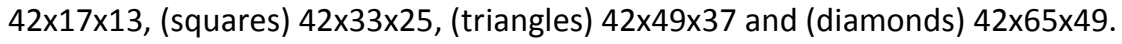

The orders of magnitude and the evolution of velocity fluctuations calculated with various computational grids agree fairly well, as shown in Fig. 14. Yet local disparities reaching almost $40 \%$ are observed. There is no evident trend that describes the evolution of the RMS with cells size. All RMS decrease in the range $43 \times 33 \times 25$ to $43 \times 65 \times 49$ without stabilizing. The highest values are obtained with the mesh $43 \times 33 \times 25$ and drop for $43 \times 17 \times 13$. Variations of the RMS, not presented here, are also observed in the HCD case with a bubble radius to cell size ratio ranging from 0.7 to 4 with no constant evolution trend neither.

The global characterization of two-phase flows can reliably be conducted by means of averaged quantities, such as the velocity and the void fraction, computed for variables meshes without major modification of the results. We would recommend being precautious in the analysis of RMS results though due to the difficulty of choosing a numerical grid in multiphase 
flows with finite size dispersed elements. We sense that computational cells should be at least various times larger than bubble radius in order to cumulate a significant amount of momentum transfer, but limited to avoid the averaging of local effects.

\section{5 - Wall shear-stress statistics}

One of the major advantages of DNS is the fine resolution of the flow in the wall vicinity, which is a crucial region for various physical phenomena. The hydrodynamic wall-shear stress is one of the interesting statistics in those cases since it can also be related to the Nusselt number through the well-known Reynolds analogy. A short study of the evolution of the velocity gradient on the wall is conducted for the HCD case and two cases with smaller bubble production rate. The set of numerical parameters are summarized in Table 5.

Table 5 : Numerical set-up for the study of wall shear rate.

\begin{tabular}{|c|c|c|c|c|c|}
\hline$\Delta \boldsymbol{t}^{*}$ & $\boldsymbol{N}_{\boldsymbol{x}} \times \boldsymbol{N}_{\boldsymbol{y}} \times \boldsymbol{N}_{\mathbf{z}}$ & $\boldsymbol{L}_{\boldsymbol{x}}^{*} \times \boldsymbol{L}_{\boldsymbol{y}}^{*} \times \boldsymbol{L}_{\mathbf{z}}^{*}$ & $\boldsymbol{r}^{*}$ & $\boldsymbol{R} \boldsymbol{e}_{\boldsymbol{h}}$ & $\dot{\boldsymbol{N}} \boldsymbol{b}_{\boldsymbol{y}^{*}=\mathbf{0}}$ \\
\hline 0.01 & $65 \times 33 \times 17$ & $16 \times 2 \times 0.94$ & 0.03 & 100 & $\dot{N} b_{y^{*}=0}=10, N b_{y^{*}=0}=5$ and \\
& & & & & $N b_{y^{*}=0}=3.3$ \\
\hline
\end{tabular}

The shear evolution presented in Fig. 15 is an illustration of the variety of information possibly reached by the DNS. It shows the non-dimensional wall shear-rate $\dot{\gamma}_{w}^{*}=\left.\frac{\partial u_{x}^{*}}{\partial y^{*}}\right|_{y=0}$ versus the non-dimensional electrode height, computed for bubble production rates of $N b_{y^{*}=0}=10$, $\dot{N} b_{y^{*}=0}=5$ and $\dot{N} b_{y^{*}=0}=3.3$. Predictably, the magnitude of the shear-rate increases with electrode height because of the bubble-induced flow acceleration. All three computed shearrates start with the Poiseuille value of $\dot{\gamma}_{w}^{*}=2$, rise rapidly at first and then more steadily until reaching the values of $10.2,8.2$ and 7.2 respectively. 


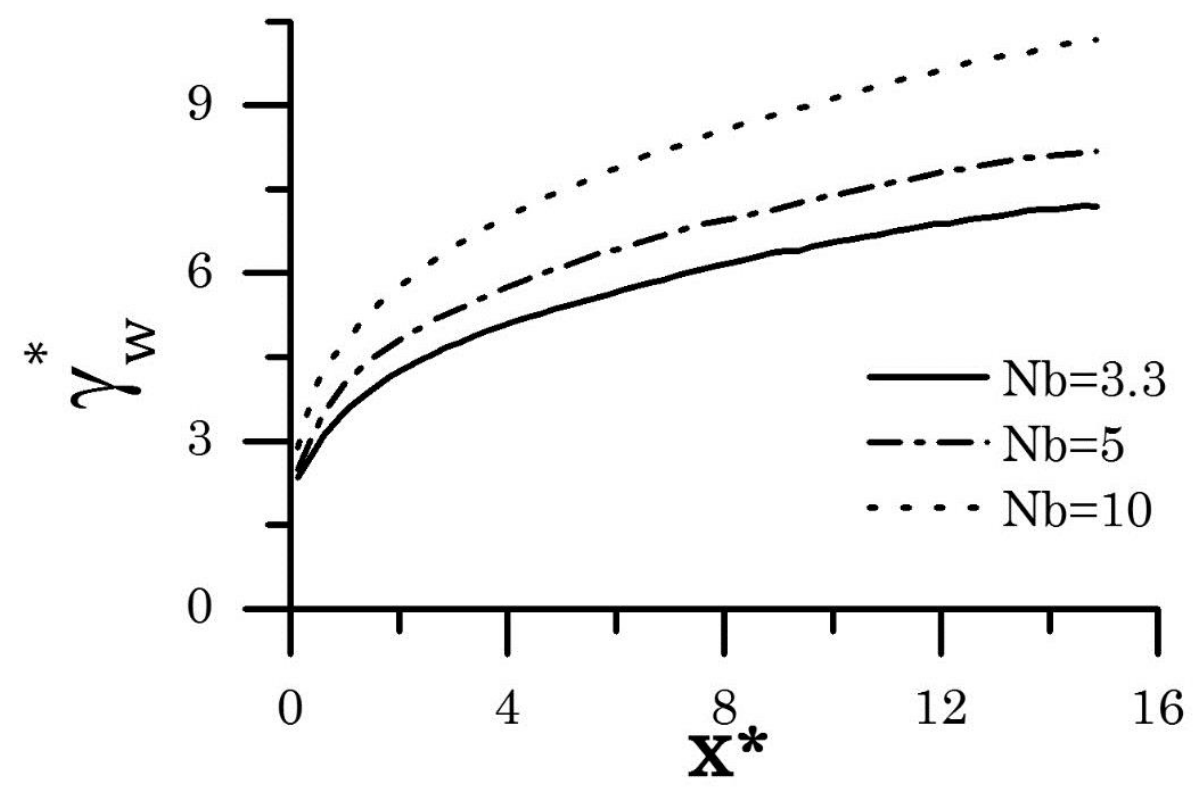

Fig. 15 : Evolution of the non-dimensional wall shear-rate versus the non-dimensional electrode height. Results are presented for (dot line) $N b_{y^{*}=0}=10$, (dash-dot line) $\dot{N} b_{y^{*}=0}=5$ and (full line) $\dot{N} b_{y^{*}=0}=3.3$.

\section{Conclusion}

This work is dedicated to the Eulerian-Lagrangian simulation of a laminar two-phase flow in a vertical channel. Bubbles dynamics and their interactions with the walls and the liquid are fully resolved through a four-way coupling strategy (i.e. taking into account bubble-fluid and bubble-bubble interactions). Configurations composed of one and two gas-evolving electrodes are treated with typical parameters found in classical electrochemically generated flows. Since void fraction takes significant values in the near wall region, simulations involve a very large amount of bubbles and may require up to 45.000 interacting bubbles in the computational domain for the two gas-evolving electrodes. However, due to the spanwise direction reduction and the bubbles collision algorithm simplification, the computational requirement is not prohibitive and all runs could be easily performed on a standard workstation via an OpenMp parallel strategy or even on a single processor in a moderate time.

DNS results are consistent with the existing experimental data and the numerical mixture model (Schillings et al., 2015). At this point, more experimental work is necessary to 
discriminate what kind of numerical model is closer to the reality, but the DNS strategy gives access to a fine evaluation of the flow in the wall vicinity that is inaccessible in mixture models. The mesh sensitivity has been investigated for cell sizes ranging between 0.7 and 12 bubble radii and results show a negligible impact on the averaged quantities such as the void fraction or the velocity. However, under laminar conditions, the mesh quality seems to have an effect on their RMS values, which are not significant here but need to be clarified for future investigations with turbulent base flow. The nature of collisions has also been investigated, and no strong differences have been observed between the purely elastic and the non-elastic rebound cases, in the present flow configurations.

Last but not least, it is worth mentioning that the DNS approach is self-consistent and does not rely on empirical collective correlations or closure relations. This is obviously an appropriate choice to investigate complex multiphase flow interactions, paving also the way for the turbulent regime. We finally believe that the present work shows how DNS, which are often restricted to academic problems, can nowadays be an efficient way to bring answers to the complex questions arising from some practical chemical engineering problems. 


\section{Acknowledgements}

Authors are grateful for the financial support of the AGIR 2013 program (Grenoble Institute of Technology) and the NEEDS French program of the National Centre for Scientific Research (CNRS). 


\section{Annexe 1: the optimum lift force}

In the region closest to the wall such that $l \ll \min \left(L_{\gamma}, L_{S}\right)$, the formulation from Cherukat and Mclaughlin (1994) is expressed as:

$$
\begin{aligned}
& \frac{\boldsymbol{F}_{\text {lift }}}{\frac{3 \rho_{C} V_{r, x}^{2}}{4 \pi r}}=\left(1.7716+0.2160 \kappa-0.7292 \kappa^{2}+0.4854 \kappa^{3}\right) \boldsymbol{n} \\
&-\left(3.2397 \kappa^{-1}+1.1450+2.0840 \kappa-0.9059 \kappa^{2}\right) \Lambda \boldsymbol{e}_{y} \\
&+\left(2.0069+1.0575 \kappa-2.4007 \kappa^{2}+1.3174 \kappa^{3}\right) \Lambda^{2} \boldsymbol{n}
\end{aligned}
$$

where $\Lambda=\frac{r \dot{\gamma}}{V_{r, x}}$ is the non-dimensional shear, $\boldsymbol{n}$ is the inner normal vector of the closest wall and $\boldsymbol{e}_{y}$ is the unity vector on $\boldsymbol{y}$ direction.

Far from the wall, the expression from McLaughlin (1993) is preferred:

$$
\boldsymbol{F}_{\text {lift }}=-\frac{27 \rho_{C}}{4 \pi^{2} r} V_{r, x} \operatorname{sign}(\dot{\gamma}) \sqrt{v_{C}|\dot{\gamma}|} J \boldsymbol{e}_{y}
$$

where the function $J$ takes into account contributions from the shear-induced lift in an unbounded flow expressed and the presence of the wall. Various authors proposed a formulation of $J$ for a certain range of $l^{\gamma}$ and $\epsilon$. For small $l^{\gamma}$ and large $|\epsilon|$, the expression from Cox and Hsu (1977) is appropriate:

$$
J=\frac{\pi^{2}}{16}\left(\frac{11}{6} l^{\gamma}-\frac{1}{\epsilon}\right)
$$

Vasseur and Cox (1977) derived an expression for small $|\epsilon|$ :

$$
J=\frac{2 \pi^{2}}{3|\epsilon|} I+J^{u}
$$

The term $I$ is an integral that can be found in the work of Vasseur and Cox (1977) and that was evaluated the approximated by Arcen et al. (2006) based on fourth order polynomials:

$$
I=-3.1 \cdot 10^{-4} l^{S^{4}}+3.53 \cdot 10^{-3} l^{S^{3}}-1.088 \cdot 10^{-2} l^{S^{2}}-9.86 \cdot 10^{-3} l^{S}+9.57 \cdot 10^{-2}
$$


when $0 \leq l^{S} \leq 4.5$,

$$
I=6.78 \cdot 10^{-7} l^{S^{4}}-4.81 \cdot 10^{-5} l^{S^{3}}+1.26 \cdot 10^{-3} l^{S^{2}}-1.485 \cdot 10^{-2} l^{S}+7.05 \cdot 10^{-2} \quad \text { (20) (b) }
$$

when $4.5<l^{S} \leq 25$ and

$$
I=0
$$

when $25<l^{S}$.

The function $J^{u}$ was derived by McLaughlin (1991) and approximated in a simpler form by Legendre and Magnaudet (1998):

$$
J^{u}=2.255\left(1+0.2 \epsilon^{-2}\right)^{-1.5}
$$

Finally, McLaughlin (1993) obtained a closed form solution which they tabulated for the range $|\epsilon| \geq 0.2$ and $0.1 \leq l^{\gamma} \leq 5$ and which was approximated by Takemura et al. (2009) for a more convenient use:

$$
J=\left[1-\exp \left(-\frac{11 \pi^{2}}{96} \frac{l^{\gamma}}{J^{u}}\right)\right] J^{u}-\frac{\pi^{2}}{\epsilon} I_{L 0} \exp \left(-0.22|\epsilon|^{0.8} l^{\gamma^{2.5}}\right)
$$

where $I_{L 0}$ stands for the transverse force coefficient in the unsheared case:

$$
I_{L 0}=\left(\frac{1}{16}+3.21 \cdot 10^{-7} l^{S^{4.58}}\right) \exp \left(-0.292 l^{S}\right)
$$

For $l^{\gamma}>5$, McLaughlin (1993) give the approximate expression of $J$ :

$$
J=J^{u}-\frac{1.879}{l^{5 / 3}}
$$

The formulation of the optimum lift force is synthetized in Table 6 with the list of equations and their range of applicability: 
Table 6 : Synthesis of the optimum lift force equations.

\begin{tabular}{|c|l|l|l|}
\hline & \multicolumn{1}{|c|}{$|\epsilon|<0.2$} & \multicolumn{1}{|c|}{$0.2 \leq|\epsilon| \leq 2$} & \multicolumn{1}{|c|}{$|\epsilon|>2$} \\
\hline$l^{\gamma}<\min \left(1, L_{S} / L_{\gamma}\right)$ & $(16)$ & $(16)$ & (16) \\
\hline $\min \left(1, L_{S} / L_{\gamma}\right) \leq l^{\gamma}<0.1$ & $(17)(19)(20)(21)$ & $(17)(18)$ & (17) (18) \\
\hline $\max \left(0.1, \min \left(1, L_{S} / L_{\gamma}\right)\right) \leq l^{\gamma} \leq 5$ & $(17)(19)(20)(21)$ & $(17)(22)(21)(23)$ & (17) (22)(21)(23) \\
\hline$l^{\gamma}>\max \left(5, L_{S} / L_{\gamma}\right)$ & $(17)(24)(21)$ & $(17)(24)(21)$ & (17) (24)(21) \\
\hline
\end{tabular}




\section{Nomenclature}

\author{
Latin symbols \\ $C_{d}$ drag coefficient \\ $C_{\|, \perp} \quad$ parallel and perpendicular drag \\ corrections \\ $D$ non-dimensional hydrodynamic diffusion \\ coefficient \\ $e$ unity vector \\ $f$ hindering function \\ $f_{\text {ext }}$ back force \\ $F$ volume force \\ $F_{C-D}$ hydrodynamic force \\ $g$ gravitational constant \\ $h$ half inter-electrode gap \\ $h_{l}$ lubrication distance \\ $i$ current density \\ I Vasseur \& Cox integral \\ $I_{L 0}$ unsheared transverse force coefficient \\ $l$ nearest wall distance \\ $l^{S}$ dimensionless Stokes wall distance \\ $l^{\gamma}$ dimensionless shear wall distance \\ J McLaughlin function \\ $L$ domain length \\ $L_{S}$ Stokes lengthscale \\ $L_{\gamma}$ Saffman lengthscale \\ $n$ normal vector \\ $N$ grid nodes number \\ $\mathrm{N} b$ bubbles per time step
}

$N_{C N}$ number of Cranck-Nicholson iterations

$p$ absolute pressure

$\nabla P$ mean pressure gradient

$\nabla p^{\prime} \quad$ pressure gradient fluctuations

$r$ bubble radius

$R$ ideal gas constant

$R e_{D} \quad$ particle Reynolds number

$R e_{h} \quad$ DNS Reynolds number

$R e_{\gamma} \quad$ shear base Reynolds number

St Stokes number

$t$ time

$T$ period

$T_{\text {pmean }}, T_{\text {pfluc }}, T_{a d v}, T_{\text {diff }}, T_{\text {fext }}$ mean

pressure, fluctuating pressure, advection, diffusion, back force NS terms

$u \quad$ continuous phase velocity

$\tilde{u}$ intermediate velocity

$u_{C P}$ maximum Poiseuille velocity

$v$ bubble velocity

$v_{\text {drift }}$ drift velocity

$v_{\text {Stokes }}$ Stokes velocity

$V^{*}$ non-dimensional velocity scale

$V_{r}$ relative velocity

$x, y, z$ streamwise, wall normal, spanwise positions

$\Delta X^{*} \quad$ non-dimensional displacement scale 
Greek symbols

$\alpha$ void fraction

$\beta$ non-dimensional shear-induced diffusion

coefficient

$\dot{\gamma}$ shear rate

$\Lambda$ non-dimensional shear

$\delta_{\alpha}$ void fraction boundary layer thickness

$\epsilon$ shear/Stokes ratio

$\varepsilon_{r}$ restitution coefficient

$\kappa$ radius to wall distance ratio

$\tau$ shear stress

$\mu$ dynamic viscosity

$v$ kinematic viscosity

$\rho$ density

$\omega$ non-dimensional shear-induced migration coefficient

Subscripts

add added mass

app approach
C continuous phase

colcollision

$D$ dispersed phase

$i, j$ relative to bubble $i, j$

ovlp overlap

reb rebound

stat stationary

$w$ wall

$x, y, z$ streamwise, wall-normal, spanwise components

$x=\cdots, y=\cdots \quad$ variable evaluation at position ...

Superscripts

* non-dimensional variable

$k$ variable evaluated at time step $\mathrm{k}$ $-t, z$ time- and $z$ - averaged variable

RMS RMS of the variable

Bold style applies for vectors 


\section{References}

Akita, K., Yoshida, F., 1974. Bubble Size, Interfacial Area, and Liquid-Phase Mass Transfer Coefficient in Bubble Columns. Ind. Eng. Chem. Process Des. Dev. 13, 84-91. doi:10.1021/i260049a016

Arcen, B., Tanière, A., Oesterlé, B., 2006. On the influence of near-wall forces in particle-laden channel flows. Int. J. Multiph. Flow 32, 1326-1339. doi:10.1016/j.ijmultiphaseflow.2006.06.009

Bauer, F., Tardu, S., Doche, O., 2015. Efficiency of high accuracy DRP schemes in direct numerical simulations of incompressible turbulent flows. Comput. Fluids 107, 123-140. doi:10.1016/j.compfluid.2014.10.009

Berg, T.H.V.D., Luther, S., Mazzitelli, I.M., Rensen, J.M., Toschi, F., Lohse, D., 2006. Turbulent bubbly flow. J. Turbul. 7, N14. doi:10.1080/14685240500460782

Boissonneau, P., Byrne, P., 2000. An experimental investigation of bubble-induced free convection in a small electrochemical cell. J. Appl. Electrochem. 30, 767-775. doi:10.1023/A:1004034807331

Boivin, M., Simonin, O., Squires, K.D., 1998. Direct numerical simulation of turbulence modulation by particles in isotropic turbulence. J. Fluid Mech. 375, 235-263.

Boussinesq, J., 1885. Sur la résistance qu'oppose un liquide indéfini en repos. CR Acad Sci Paris 100, 935-937.

Brenner, H., 1961. The slow motion of a sphere through a viscous fluid towards a plane surface. Chem. Eng. Sci. 16, 242-251. doi:10.1016/0009-2509(61)80035-3

Cherukat, P., Mclaughlin, J.B., 1994. The inertial lift on a rigid sphere in a linear shear flow field near a flat wall. J. Fluid Mech. 263, 1-18. doi:10.1017/S0022112094004015

Cox, R.G., Hsu, S.K., 1977. The lateral migration of solid particles in a laminar flow near a plane. Int. J. Multiph. Flow 3, 201-222. doi:10.1016/0301-9322(77)90001-5

Dahlkild, A.A., 2001. Modelling the two-phase flow and current distribution along a vertical gasevolving electrode. J. Fluid Mech. 428, 249-272. doi:10.1017/S0022112000002639

Davis, R.H., Serayssol, J.-M., Hinch, E.J., 1986. The elastohydrodynamic collision of two spheres. J. Fluid Mech. 163, 479-497.

Delnoij, E., Lammers, F.A., Kuipers, J.A.M., van Swaaij, W.P.M., 1997. Dynamic simulation of dispersed gas-liquid two-phase flow using a discrete bubble model. Chem. Eng. Sci. 52, 1429-1458. doi:10.1016/S0009-2509(96)00515-5

Doche, O., Bauer, F., Tardu, S., 2013. Direct numerical simulations of electrochemical reactions in turbulent flow. Electrochimica Acta 88, 365-372. doi:10.1016/j.electacta.2012.09.098

Doche, O., Bauer, F., Tardu, S., 2012. Direct Numerical Simulation of an electrolyte deposition under a turbulent flow - A first approach. J. Electroanal. Chem. 664, 1-6. doi:10.1016/j.jelechem.2011.10.003

Domgin, J., Huilier, D., Karl, J., Gardin, P., Burnage, H., 1998. 'Experimental and Numerical Study of Rigid Particles, Droplets, and Bubbles Motion in Quiescent and Turbulent FlowsInfluence of the History Force, in: Third International Conference on Multiphase Flow, ICMF-98, Lyon, France. 
Druzhinin, O.A., Elghobashi, S.E., 2001. Direct numerical simulation of a three-dimensional spatially developing bubble-laden mixing layer with two-way coupling. J. Fluid Mech. 429, 23-61. doi:10.1017/S0022112000002603

Esmaeeli, A., Tryggvason, G., 1999. Direct numerical simulations of bubbly flows Part 2. Moderate Reynolds number arrays. J. Fluid Mech. 385, 325-358. doi:10.1017/S0022112099004310

Faxén, H., 1923. Die Bewegung einer starren Kugel längs der Achse eines mit zäher Flüssigkeit gefüllten Rohres. Ark. Foer Mat. Astron. Och Fys. 17, 1-28.

Gatignol, R., 1983. The Faxén formulas for a rigid particle in an unsteady non-uniform Stokesflow. J. Mécanique Théorique Appliquée 2, 143-160.

Gondret, P., Lance, M., Petit, L., 2002. Bouncing motion of spherical particles in fluids. Phys. Fluids 1994-Present 14, 643-652. doi:10.1063/1.1427920

Hocking, L.M., 1973. The effect of slip on the motion of a sphere close to a wall and of two adjacent spheres. J. Eng. Math. 7, 207-221. doi:10.1007/BF01535282

Hoomans, B.P.B., Kuipers, J.A.M., Briels, W.J., van Swaaij, W.P.M., 1996. Discrete particle simulation of bubble and slug formation in a two-dimensional gas-fluidised bed: A hardsphere approach. Chem. Eng. Sci. 51, 99-118. doi:10.1016/0009-2509(95)00271-5

Ishii, M., 1987. Interfacial Area Modeling, Vol. 3. McGraw-Hill, New York.

Janssen, L.J.J., Sillen, C.W.M.P., Barendrecht, E., van Stralen, S.J.D., 1984. Bubble behaviour during oxygen and hydrogen evolution at transparent electrodes in $\mathrm{KOH}$ solution. Electrochimica Acta 29, 633-642. doi:10.1016/0013-4686(84)87122-4

Jiménez, J., 2003. Computing high-Reynolds-number turbulence: will simulations ever replace experiments?*. J. Turbul. 4, 1-14.

Laín, S., Bröder, D., Sommerfeld, M., Göz, M.F., 2002. Modelling hydrodynamics and turbulence in a bubble column using the Euler-Lagrange procedure. Int. J. Multiph. Flow 28, 13811407. doi:10.1016/S0301-9322(02)00028-9

Lapin, A., Lübbert, A., 1994. Numerical simulation of the dynamics of two-phase gas-liquid flows in bubble columns. Chem. Eng. Sci. 49, 3661-3674. doi:10.1016/00092509(94)E0121-6

Legendre, D., Magnaudet, J., 1998. The lift force on a spherical bubble in a viscous linear shear flow. J. Fluid Mech. 368, 81-126. doi:10.1017/S0022112098001621

Leighton, D., Acrivos, A., 1987. Measurement of shear-induced self-diffusion in concentrated suspensions of spheres. J. Fluid Mech. 177, 109-131. doi:10.1017/S0022112087000880

Lu, J., Biswas, S., Tryggvason, G., 2006. A DNS study of laminar bubbly flows in a vertical channel. Int. J. Multiph. Flow 32, 643-660. doi:10.1016/j.ijmultiphaseflow.2006.02.003

Maxey, M.R., Riley, J.J., 1983. Equation of motion for a small rigid sphere in a nonuniform flow. Phys. Fluids 1958-1988 26, 883-889. doi:10.1063/1.864230

Mazzitelli, I.M., Lohse, D., Toschi, F., 2003. The effect of microbubbles on developed turbulence. Phys. Fluids 1994-Present 15, L5-L8. doi:10.1063/1.1528619

McLaughlin, J.B., 1993. The lift on a small sphere in wall-bounded linear shear flows. J. Fluid Mech. 246, 249-265. doi:10.1017/S0022112093000114

McLaughlin, J.B., 1991. Inertial migration of a small sphere in linear shear flows. J. Fluid Mech. 224, 261-274. 
Nicolai, H., Herzhaft, B., Hinch, E.J., Oger, L., Guazzelli, E., 1995. Particle velocity fluctuations and hydrodynamic self-diffusion of sedimenting non-Brownian spheres. Phys. Fluids 1994Present 7, 12-23. doi:10.1063/1.868733

Nierhaus, T., Vanden Abeele, D., Deconinck, H., 2007. Direct numerical simulation of bubbly flow in the turbulent boundary layer of a horizontal parallel plate electrochemical reactor. Int. J. Heat Fluid Flow 28, 542-551. doi:10.1016/j.ijheatfluidflow.2007.03.005

Oseen, C., 1910. Ueber die stokes' sche formel, und iiber eine venvandt. e aufgabe in der hydrodynamik. Ark Math Astron. Fys 6.

Saffman, P.G., 1965. The lift on a small sphere in a slow shear flow. J. Fluid Mech. 22, 385-400. doi:10.1017/S0022112065000824

Schiller, L., Naumann, A., 1935. A drag coefficient correlation. Vdi Ztg. 77, 51.

Schillings, J., Doche, O., Deseure, J., 2015. Modeling of electrochemically generated bubbly flow under buoyancy-driven and forced convection. Int. J. Heat Mass Transf. 85, 292-299. doi:10.1016/j.ijheatmasstransfer.2015.01.121

Squires, K.D., Eaton, J.K., 1990. Particle response and turbulence modification in isotropic turbulence. Phys. Fluids Fluid Dyn. 1989-1993 2, 1191-1203. doi:10.1063/1.857620

Takemura, F., Magnaudet, J., Dimitrakopoulos, P., 2009. Migration and deformation of bubbles rising in a wall-bounded shear flow at finite Reynolds number. J. Fluid Mech. 634, 463486. doi:10.1017/S0022112009007605

Tam, C.K.W., Webb, J.C., 1993. Dispersion-Relation-Preserving Finite Difference Schemes for Computational Acoustics. J. Comput. Phys. 107, 262-281. doi:10.1006/jcph.1993.1142

Tryggvason, G., Bunner, B., Esmaeeli, A., Juric, D., Al-Rawahi, N., Tauber, W., Han, J., Nas, S., Jan, Y.-J., 2001. A Front-Tracking Method for the Computations of Multiphase Flow. J. Comput. Phys. 169, 708-759. doi:10.1006/jcph.2001.6726

Vasseur, P., Cox, R.G., 1977. The lateral migration of spherical particles sedimenting in a stagnant bounded fluid. J. Fluid Mech. 80, 561-591. doi:10.1017/S0022112077001840

Wakiya, S., 1960. Research report 9. Fac. Eng. Niigata Univ. Jpn.

Wang, Q., Squires, K.D., Chen, M., McLaughlin, J.B., 1997. On the role of the lift force in turbulence simulations of particle deposition. Int. J. Multiph. Flow 23, 749-763. doi:10.1016/S0301-9322(97)00014-1

Zhang, D.Z., Prosperetti, A., 1994. Ensemble phase-averaged equations for bubbly flows. Phys. Fluids 1994-Present 6, 2956-2970. doi:10.1063/1.868122 